The Annals of Applied Probability

2012, Vol. 22, No. 1, 239-284

DOI: $10.1214 / 11-A A P 767$

(C) Institute of Mathematical Statistics, 2012

\title{
PORTFOLIOS AND RISK PREMIA FOR THE LONG RUN ${ }^{1}$
}

\author{
By Paolo Guasoni and Scott Robertson \\ Boston University, Dublin City University and Carnegie Mellon University
}

This paper develops a method to derive optimal portfolios and risk premia explicitly in a general diffusion model for an investor with power utility and a long horizon. The market has several risky assets and is potentially incomplete. Investment opportunities are driven by, and partially correlated with, state variables which follow an autonomous diffusion. The framework nests models of stochastic interest rates, return predictability, stochastic volatility and correlation risk.

In models with several assets and a single state variable, long-run portfolios and risk premia admit explicit formulas up the solution of an ordinary differential equation which characterizes the principal eigenvalue of an elliptic operator. Multiple state variables lead to a quasilinear partial differential equation which is solvable for many models of interest.

The paper derives the long-run optimal portfolio and the long-run optimal pricing measures depending on relative risk aversion, as well as their finite-horizon performance.

Introduction. Long-run asymptotics are a powerful tool to obtain explicit formulas in portfolio choice and derivatives pricing but their use has been mostly ad hoc in the absence of general results. This paper develops a method to derive optimal portfolios and risk premia explicitly in a general diffusion model for an investor with power utility and in the limit of a long horizon. The market has several risky assets and is potentially incomplete. Investment opportunities are driven by, and partially correlated with, state variables that follow an autonomous diffusion.

Investment and pricing problems share a reputation for mathematical complexity. This common trait is not an accident; the central message of

\footnotetext{
Received July 2009; revised February 2011.

${ }^{1}$ Supported in part by NSF (DMS-053239 and DMS-0807994), SFI (07/MI/008, 07/SK/M1189, 08/SRC/FMC1389) and the European Commission (RG-248896).

AMS 2000 subject classifications. Primary 91G10, 62P05; secondary 91G20.

Key words and phrases. Long-run, portfolio choice, derivatives pricing, incomplete markets.
}

This is an electronic reprint of the original article published by the Institute of Mathematical Statistics in The Annals of Applied Probability, 2012, Vol. 22, No. 1, 239-284. This reprint differs from the original in pagination and typographic detail. 
duality theory ${ }^{2}$ is that the two problems are indeed equivalent, as state-price densities are proportional to the marginal utilities of optimal payoffs. In spite of this conceptual equivalence, portfolio choice and derivatives pricing have followed largely different strands of literature, each of them with its own terminology.

The portfolio choice literature focuses on finding the intertemporal hedging component of optimal portfolios. ${ }^{3}$ Long-run asymptotics have appeared in this literature under different names: the risk-sensitive control approach, ${ }^{4}$ turnpike results ${ }^{5}$ and large deviations criteria $^{6}$ are all efforts to achieve tractability by means of the long-run limit.

The derivatives pricing literature strives to identify martingale measures that are optimal in the sense of the minimax martingale measure of $\mathrm{He}$ and Pearson (1991) or the least favorable completion of Karatzas et al. (1991). Power utility leads to the $q$-optimal measure ((Hobson, 2004), (Henderson, 2005)) which embeds several other martingale measures; ${ }^{7}$ it reduces to the minimal measure for $q=0$, to the minimal entropy measure for $q=1$ and to the variance-optimal measure for $q=2$.

The advantages of long-run asymptotics are their tractability and accuracy. Long-run portfolios and risk premia are much simpler than their finite-horizon counterparts and allow explicit expressions even in cases in which the latter do not. In general, long-run policies are identified by the quasilinear partial differential equation (22) which admits explicit solutions in several models of interest. In the case of a single state variable, this equation reduces to an ordinary differential equation, which is furthermore linear if the state variable has a constant correlation with excess returns. The accuracy of the long-run approach stems from the bounds (27) which estimate the duality gap at any horizon and hence the potential departure from the unknown finite-horizon optimum. Long-run optimality holds (Definition 6) when long-run policies are approximately optimal over long horizons. The

\footnotetext{
${ }^{2}$ See, for example, Pliska (1986), Karatzas, Lehoczky and Shreve (1987), Cox and Huang (1989), He and Pearson (1991), Kramkov and Schachermayer (1999), as well as CastañedaLeyva and Hernández-Hernández (2005) for a setting similar to the one in this paper.

${ }^{3}$ Kim and Omberg (1996), Brennan and Xia (2002), Wachter (2002), Munk and Sørensen (2004), Liu (2007), compute optimal portfolios explicitly for certain models.

${ }^{4}$ Fleming and McEneaney (1995), Nagai (1996, 2003), Bielecki and Pliska (1999, 2000), Fleming and Sheu (2000, 2002), Kuroda and Nagai (2002) and Nagai and Peng (2002), Hata and Sekine (2005), Kaise and Sheu (2004, 2006).

${ }^{5}$ Leland (1972), Hakansson (1974), Huberman and Ross (1983), Cox and Huang (1992), Jin (1998), Huang and Zariphopoulou (1999), Dybvig, Rogers and Back (1999).

${ }^{6}$ Pham (2003), Föllmer and Schachermayer (2007).

${ }^{7}$ Logarithmic utility leads to the minimal measure used by Föllmer and Schweizer (1991). Exponential utility leads to the minimal-entropy measure Grandits and Rheinländer (2002), Frittelli (2000), Rheinländer (2005). Mean-variance hedging leads to the variance-optimal measure introduced by Schweizer $(1992,1996)$.
} 
main result of this paper gives a sufficient condition for long-run optimality in a general multidimensional diffusion. Furthermore, this condition is sharp for certain models and a calibration to the parameters estimated by Barberis (2000) shows that it is satisfied for reasonable levels of risk aversion.

Two duality insights are central to our results. First, the usual duality between payoffs and martingale densities extends to their stochastic logarithms, which are portfolios and risk premia. Second, long-run asymptotics become easier in a duality context because candidate long-run risk-premia yield an upper bound on the maximal expected utility and vice versa. This observation allows to overcome some difficulties arising in the verification theorems of the risk-sensitive control literature.

An important concept arising in long-run analysis is the myopic probability; a long-run investor with power utility under the original probability behaves like a logarithmic (or myopic) investor under the myopic probability. This probability plays an important role both for long-run analysis and for finitehorizon bounds and its existence is crucial for the long-run optimality result.

The rest of the paper is organized as follows. Section 1 describes the model in detail, introducing notation. Section 2 contains the main result: a general method to obtain long-run policies in closed form. It also provides sufficient conditions, adapted from Kaise and Sheu (2006), for the existence of solutions to the associated ergodic Bellman equation. Section 3 discusses the various implications of these results for portfolio choice and derivatives pricing, and the connections with the stochastic control and large deviations approaches. Section 4 derives long-run portfolios and risk premia in two models of interest. The last one combines stochastic interest rates, drifts and volatilities, and still admits simple closed form solutions. Section 5 concludes. All proofs are in the appendices.

\section{Model.}

1.1. Market. Consider a financial market with a risk-free asset $S^{0}$ and $n$ risky assets $S=\left(S^{1}, \ldots, S^{n}\right)$. Investment opportunities (interest rates, expected returns and covariances) depend on $k$ state variables $Y=\left(Y^{1}, \ldots, Y^{k}\right)$ which model their change over time,

$$
\begin{aligned}
& \frac{d S_{t}^{0}}{S_{t}^{0}}=r\left(Y_{t}\right) d t \\
& \frac{d S_{t}^{i}}{S_{t}^{i}}=r\left(Y_{t}\right) d t+d R_{t}^{i}, \quad 1 \leq i \leq n .
\end{aligned}
$$

Cumulative excess returns $R=\left(R^{1}, \ldots, R^{n}\right)$ and state variables follow the diffusion

$$
d R_{t}^{i}=\mu_{i}\left(Y_{t}\right) d t+\sum_{j=1}^{n} \sigma_{i j}\left(Y_{t}\right) d Z_{t}^{j}, \quad 1 \leq i \leq n
$$




$$
\begin{gathered}
d Y_{t}^{i}=b_{i}\left(Y_{t}\right) d t+\sum_{j=1}^{k} a_{i j}\left(Y_{t}\right) d W_{t}^{j}, \quad 1 \leq i \leq k, \\
d\left\langle Z^{i}, W^{j}\right\rangle_{t}=\rho_{i j}\left(Y_{t}\right) d t, \quad 1 \leq i \leq n, 1 \leq j \leq k,
\end{gathered}
$$

where $Z=\left(Z^{1}, \ldots, Z^{n}\right)$ and $W=\left(W^{1}, \ldots, W^{k}\right)$ are multivariate Brownian motions. This setting provides a flexible framework that nests most diffusion models in finance, including the models of correlation risk considered by Buraschi, Porchia and Trojani (2010) in which $\rho$ is a function of a state variable.

The law of $(R, Y)$ determines the drifts $b, \mu$ and the covariation matrices $\Sigma=\sigma \sigma^{\prime}=d\langle R, R\rangle_{t} / d t, A=a a^{\prime}=d\langle Y, Y\rangle_{t} / d t$ and $\Upsilon=\sigma \rho a^{\prime}=d\langle R, Y\rangle_{t} / d t$, where the prime sign denotes matrix transposition. By contrast, the matrices $\sigma, a, \rho$ are identified only up to orthogonal transformations. The market defined by (1)-(5) is in general incomplete and the covariance matrix $\Upsilon^{\prime} \Sigma^{-1} \Upsilon$ gauges the degree of incompleteness of the market, highlighting two extremes: complete markets for $\Upsilon^{\prime} \Sigma^{-1} \Upsilon=A$ and fully incomplete markets for $\Upsilon=0$.

Let $E \subseteq \mathbb{R}^{k}$ be an open connected set. Denote by $C^{m}\left(E, \mathbb{R}^{d}\right)\left[\right.$ resp., $C^{m, \gamma}(E$, $\left.\mathbb{R}^{d}\right)$ ] the class of $\mathbb{R}^{d}$-valued functions on $E$ with continuous (resp., locally $\gamma$-Hölder continuous) partial derivatives of $m$ th order. The superscripts are dropped for $m=0$ or $d=1$, so that $C^{0, \gamma}\left(E, \mathbb{R}^{1}\right)$ is denoted by $C^{\gamma}(E, \mathbb{R})$. The following assumption prescribes that the coefficients $r, \mu, b, A, \Sigma$ and $\Upsilon$ are regular and nondegenerate.

Assumption 1. $r \in C^{\gamma}(E, \mathbb{R}), b \in C^{1, \gamma}\left(E, \mathbb{R}^{k}\right), \mu \in C^{1, \gamma}\left(E, \mathbb{R}^{n}\right), \quad A \in$ $C^{2, \gamma}\left(E, \mathbb{R}^{k \times k}\right), \Sigma \in C^{2, \gamma}\left(E, \mathbb{R}^{n \times n}\right)$ and $\Upsilon \in C^{2, \gamma}\left(E, \mathbb{R}^{n \times k}\right)$. The symmetric matrices $A$ and $\Sigma$ are positive definite for all $y \in E$.

The region $E$ is typically of the form $E=\mathbb{R}^{d} \times(0, \infty)^{k-d}$ for some $0 \leq$ $d \leq k$, as in the case of multivariate Ornstein-Uhlenbeck processes, Feller diffusions or a combination thereof. Fernholz and Karatzas (2005) consider models in which $E$ is the open simplex in $\mathbb{R}^{n-1}$.

To construct the processes $(R, Y)$, let $\Omega=C\left([0, \infty), \mathbb{R}^{n+k}\right)$ endowed with the topology of uniform convergence on bounded intervals. Let $\mathcal{B}$ be the Borel sigma algebra and let $\left(\mathcal{B}_{t}\right)_{t>0}$ be the filtration generated by the coordinate process $X$ defined by $X_{t}(\omega)=\omega_{t}$ for $\omega \in \Omega$. For a second-order differential operator $L$ of the form in (6) below, a solution to the martingale problem for $L$ on $\mathbb{R}^{n} \times E$ is a family of Borel probability measures $\left(P^{x}\right)_{x \in \mathbb{R}^{n} \times E}$ on $(\Omega, \mathcal{B})$ such that, for each $x \in \mathbb{R}^{n} \times E$, (i) $P^{x}\left(X_{0}=x\right)=1$, (ii) $P^{x}\left(X_{t} \in\right.$ $\left.\mathbb{R}^{n} \times E, \forall t \geq 0\right)=1$ and (iii) $\left(f\left(X_{t}\right)-f\left(X_{0}\right)-\int_{0}^{t}(L f)\left(X_{u}\right) d u ; \mathcal{B}_{t}\right)$ is a $P^{x}$ martingale for all $f \in C_{0}^{2}\left(\mathbb{R}^{n} \times E\right)$.

The next assumption ensures that $\mu, b, A, \Sigma$ and $\Upsilon$ identify the law of $(R, Y) . x=(r, y)$, with $r \in \mathbb{R}^{n}, y \in E$, denotes the starting points of $R$ and $Y$. 
Assumption 2. There exists a unique solution $\left(P^{(r, y)}\right)_{r \in \mathbb{R}^{n}, y \in E}$ to the martingale problem for

$$
\begin{aligned}
& L=\frac{1}{2} \sum_{i, j=1}^{n+k} \tilde{A}^{i, j}(x) \frac{\partial^{2}}{\partial x_{i} \partial x_{j}}+\sum_{i=1}^{n+k} \tilde{b}^{i}(x) \frac{\partial}{\partial x_{i}}, \\
& \tilde{A}=\left(\begin{array}{cc}
\Sigma & \Upsilon \\
\Upsilon^{\prime} & A
\end{array}\right), \quad \tilde{b}=\left(\begin{array}{c}
\mu \\
b
\end{array}\right) .
\end{aligned}
$$

Since $R_{0}=0$ for all the models considered in this paper, the measure $P^{(0, y)}$ in Assumption 2 is simply denoted as $P^{y}$. Henceforth, consider the filtration $\left(\mathcal{F}_{t}\right)_{t \geq 0}$ defined as $\mathcal{F}_{t}=\mathcal{B}_{t+}$, that is, the right continuous envelope of $\mathcal{B}_{t}$. Under Assumption 1 for $f \in C_{0}^{2}\left(\mathbb{R}^{n} \times E\right), f\left(X_{t}\right)-f\left(X_{0}\right)-\int_{0}^{t}(L f)\left(X_{u}\right) d u$ is a martingale also with respect to $\mathcal{F}_{t}$; cf. Karatzas and Shreve (1991), Section 5.4.

REMARK 3. For consistency of notation, Assumption 2 involves the joint diffusion process $(R, Y)$. However, it is essentially an assumption on the process $Y$. Indeed, if Assumption 1 holds, and if there is a unique solution $\left(Q^{y}\right)_{y \in E}$ to the martingale problem for the operator

$$
L^{Y}=\frac{1}{2} \sum_{i, j=1}^{k} A^{i, j}(y) \frac{\partial^{2}}{\partial y_{i} \partial y_{j}}+\sum_{i=1}^{k} b^{i}(y) \frac{\partial}{\partial y_{i}},
$$

then Assumption 2 holds. To see this fact, first consider the martingale problem for the operator associated to $(Y, B)$, where $B$ is an $n$-dimensional standard Brownian motion independent of $Y$. Then, write $Z=\rho W+\bar{\rho} B$ where $\bar{\rho}$ is a square root of $1_{n}-\rho \rho^{\prime}$ and define the integrals for $R$ in (3) accordingly.

1.2. Trading strategies. An investor trades in the market according to a portfolio $\pi=\left(\pi_{t}^{i}\right)_{t \geq 0}^{1 \leq i \leq n}$, representing the proportions of wealth in each risky asset. Since the investor observes the state variables $Y$ and the asset returns $R$, the portfolio $\pi$ is adapted to the filtration generated by $(R, Y)$, and is $R$-integrable. ${ }^{8}$ The corresponding wealth process $X^{\pi}=\left(X_{t}^{\pi}\right)_{t \geq 0}$ follows:

$$
\frac{d X_{t}^{\pi}}{X_{t}^{\pi}}=r\left(Y_{t}\right) d t+\pi_{t}^{\prime} d R_{t}
$$

Note that a positive initial capital $X_{0} \geq 0$ implies a positive wealth at all times, that is, $X_{t}^{\pi} \geq 0$ a.s. for all $t \geq 0$, thereby ruling out doubling strategies [see, e.g., Harrison and Pliska (1981)].

\footnotetext{
${ }^{8} R$-integrability means each of the integrals $\int_{0}^{\cdot} \pi^{\prime} \mu d t, \int_{0}^{\cdot} \pi^{\prime} \sigma d Z_{t}$ is well defined.
} 
1.3. Preferences. The investor's preferences are modeled by the power utility function

$$
U(x)=\frac{x^{p}}{p}, \quad p<1, p \neq 0 .
$$

Denoting by $E_{P}^{y}$ the expectation with respect to $P^{y}$, the goal is to maximize expected utility from terminal wealth. With a finite horizon $T$, the problem is

$$
\max _{\pi} \frac{1}{p} E_{P}^{y}\left[\left(X_{T}^{\pi}\right)^{p}\right]
$$

Since power utility is homothetic $\left[U(c x)=c^{p} U(x)\right]$, it suffices to consider the case $X_{0}=1$ of unit initial wealth. Henceforth, $q$ denotes the conjugate exponent to $p$,

$$
q:=\frac{p}{p-1}
$$

To preserve economic intuition, recall that $p=1-\gamma$ and $q=1-\frac{1}{\gamma}$, where $\gamma$ is the investor's relative risk aversion. Risk aversion increases as $q$ increases and as $p$ decreases, and logarithmic utility corresponds to the limit $p \rightarrow 0$.

The martingale approach to utility maximization relies on the duality between final payoffs and pricing rules, represented by the related concepts of stochastic discount factors and martingale measures.

Definition 4. A stochastic discount factor is a strictly positive adapted process $M=\left(M_{t}\right)_{t \geq 0}$, such that $M S$ is a martingale

$$
E_{P}^{y}\left[M_{t} S_{t}^{i} \mid \mathcal{F}_{s}\right]=M_{s} S_{s}^{i} \quad \text { for all } 0 \leq s \leq t, 0 \leq i \leq n .
$$

A martingale measure is a probability $Q$, such that $\left.Q\right|_{\mathcal{F}_{t}}$ and $\left.P^{y}\right|_{\mathcal{F}_{t}}$ are equivalent for all $t \in[0, \infty)$ and the discounted prices $S^{i} / S^{0}$ (or equivalently, the excess returns $R^{i}$ ) are $Q$-martingales for all $1 \leq i \leq n$.

Martingale measures and stochastic discount factors are in a one-to-one correspondence through the relation $\left.\frac{d Q}{d P^{y}}\right|_{\mathcal{F}_{t}}=S_{t}^{0} M_{t}$, although their distinction is important in the present context of stochastic interest rates. Except for a complete market in which the martingale measure is unique, the description of a pricing rule requires the choice of unhedgeable risk premia $\eta$. For any $\left(\mathcal{F}_{t}\right)_{t \geq 0}$ adapted, integrable process $\eta$, a candidate (local) martingale measure $Q^{\eta, y}$ is given by $d Q^{\eta, y} /\left.d P^{y}\right|_{\mathcal{F}_{t}}=Z_{t}^{\eta}$, where

$$
Z_{t}^{\eta}=\mathcal{E}\left(-\int_{0}^{\cdot}\left(\mu^{\prime} \Sigma^{-1}+\eta^{\prime} \Upsilon^{\prime} \Sigma^{-1}\right) \sigma d Z+\int_{0}^{\cdot} \eta^{\prime} a d W\right)_{t}
$$

and where $\mathcal{E}(X)_{t}=\exp \left(X_{t}-\frac{1}{2}\langle X\rangle_{t}\right)$. Clearly, $Z$ must be a martingale for $Q^{\eta, y}$ to be an equivalent local martingale measure. For such a $\eta$, let $M^{\eta}$ denote 
the corresponding stochastic discount factor

$$
M_{t}^{\eta}=\exp \left(-\int_{0}^{t} r d t\right) \mathcal{E}\left(-\int_{0}^{\cdot}\left(\mu^{\prime} \Sigma^{-1}+\eta^{\prime} \Upsilon^{\prime} \Sigma^{-1}\right) \sigma d Z+\int_{0}^{\cdot} \eta^{\prime} a d W\right)_{t} .
$$

Note that for any strategy $\pi$ and risk premia $\eta$, by (8), the process $X^{\pi} M^{\eta}$ is a super-martingale, even if the right-hand side of (12) is only a local martingale. For power utility, the following lemma applied to $X=X_{T}^{\pi}$ and $M=M_{T}^{\eta}$ for any $T>0$ shows that the duality bound is an immediate consequence of Hölder's inequality and of the super-martingale property of the process $X^{\pi} M^{\eta}$.

Lemma 5. Let $X, M$ be random variables on a probability space $(\Omega, \mathcal{F}, P)$ such that $X, M>0 P$-almost surely and $E_{P}[X M] \leq 1$. Then

$$
\frac{1}{p} E_{P}\left[X^{p}\right] \leq \frac{1}{p} E_{P}\left[M^{q}\right]^{1-p}
$$

and equality holds if and only if $E_{P}[X M]=1$ and, for some $\alpha>0$,

$$
X^{p-1}=\alpha M \text {. }
$$

Equation (14) bounds the utility of any terminal wealth by a moment of any stochastic discount factor and vice versa. The first-order condition (15) is the usual alignment of marginal utilities with state-price densities.

Consider a finite horizon $T$. Lemma 5 implies that a pair $\left(\pi^{T}, \eta^{T}\right)$ of a portfolio $\pi^{T}$ and risk premia $\eta^{T}$ such that $X=X_{T}^{\pi^{T}}$ and $M=M_{T}^{\eta^{T}}$ is optimal if it satisfies $(15)$ and $E_{P}^{y}\left[X_{T}^{\pi^{T}} M_{T}^{\eta^{T}}\right]=1$. Denoting by $u_{T}(y)$ the value function, that is, the maximal expected utility, the following equalities hold:

$$
\frac{1}{p} E_{P}^{y}\left[\left(X_{T}^{\pi^{T}}\right)^{p}\right]=u_{T}(y)=\frac{1}{p} E_{P}^{y}\left[\left(M_{T}^{\eta^{T}}\right)^{q}\right]^{1-p}
$$

hence, $\pi^{T}$ is the optimal portfolio and the stochastic discount factor $M^{\eta^{T}}$ identifies the pricing rule that makes an investor indifferent between buying and selling a small amount of any payoff, including unhedgeable ones.

1.4. Long-run optimality. In the Markov model defined by (1)-(5), stochastic control arguments [see, e.g., Pham (2002), among many others], show that the pair $\left(\pi^{T}, \eta^{T}\right)$ achieving optimality is of the form $\pi_{t}^{T}=\pi^{T}\left(T-t, Y_{t}\right)$ and $\eta_{t}^{T}=\eta^{T}\left(T-t, Y_{t}\right)$ for deterministic functions

$$
\pi^{T}:[0, T] \times E \mapsto \mathbb{R}^{n} \quad \eta^{T}:[0, T] \times E \mapsto \mathbb{R}^{k} .
$$

Thus, optimal policies depend on both state variables and the residual horizon. This joint dependence is the major source of intractability in portfolio choice and derivatives pricing problems. 
Brandt (1999), Barberis (2000) and Wachter (2002) report that optimal policies converge rapidly to functions of state variables alone. Thus the optimal policy for a long horizon $[0, T]$ is approximately equal to a timehomogeneous function for most of the interval, departing from it as the horizon $T$ approaches. The question is whether using a time-homogenous policy throughout the interval $[0, T]$ can be approximately optimal.

For any functions $\pi \in C\left(E, \mathbb{R}^{n}\right), \eta \in C\left(E, \mathbb{R}^{k}\right)$ consider the portfolio $\pi=$ $\left(\pi\left(Y_{t}\right)\right)_{t \geq 0}$ and risk premia $\eta=\left(\eta\left(Y_{t}\right)\right)_{t \geq 0}$. At any finite horizon $T$, the duality bound (14) implies that

$$
\frac{1}{p} E_{P}^{y}\left[\left(X_{T}^{\pi}\right)^{p}\right] \leq u_{T}(y) \leq \frac{1}{p} E_{P}^{y}\left[\left(M_{T}^{\eta}\right)^{q}\right]^{1-p} .
$$

The first inequality reflects the potential gap between the utility of the longrun portfolio and the value function. A tangible measure of this gap is the increase in the risk-free rate $l_{T}$ required to recover this loss, as to match the expected utility of the long-run optimal portfolio under the higher rate with the maximum expected utility at the regular rate. This is the certainty equivalent loss, defined as

$$
\frac{1}{p} E_{P}^{y}\left[\left(e^{l_{T} T} X_{T}^{\pi}\right)^{p}\right]=u_{T}(y) .
$$

Substituting (18) into (17) yields an upper bound on $l_{T}$

$$
l_{T} \leq \frac{1}{p}\left(\frac{1}{T} \log E_{P}^{y}\left[\left(M_{T}^{\eta}\right)^{q}\right]^{1-p}-\frac{1}{T} \log E_{P}^{y}\left[\left(X_{T}^{\pi}\right)^{p}\right]\right) .
$$

This argument motivates the definition of a pair $(\pi, \eta)$ as long-run optimal when its certainty equivalent loss vanishes for long horizons.

Definition 6. A pair $(\pi, \eta) \in C\left(E, \mathbb{R}^{n}\right) \times C\left(E, \mathbb{R}^{k}\right)$ is long-run optimal if, for all $y \in E$,

$$
\limsup _{T \rightarrow \infty} \frac{1}{p}\left(\frac{1}{T} \log E_{P}^{y}\left[\left(M_{T}^{\eta}\right)^{q}\right]^{1-p}-\frac{1}{T} \log E_{P}^{y}\left[\left(X_{T}^{\pi}\right)^{p}\right]\right)=0 .
$$

Long-run optimality defined here is essentially equivalent to the criterion used by Grossman and Vila (1992) to solve portfolio choice problems with leverage constraints. Grossman and Zhou (1993) apply the same idea to drawdown constraints and Dumas and Luciano (1991) to transaction costs. The risk-sensitive control literature studies a similar objective for multidimensional linear diffusions.

Definition 6 departs from the existing literature by examining both the primal (investment) and the dual (pricing) problems. This perspective yields verification theorems that are valid for general multidimensional diffusions, 
provides estimates on finite-horizon performance and allows to identify the parameter sets for which long-run optimality holds.

Definition 6 allows another interpretation based on management fees: an investor with sufficiently long horizon prefers a long-run optimal portfolio to the optimal finite-horizon portfolio if the long-run portfolio has slightly lower fees. The argument is straightforward: suppose that the portfolio $\pi$ requires the payment of a (continuously compounded) fee $\varphi$, while the finite-horizon portfolio $\pi_{T}$ entails a higher fee $\varphi_{T} \geq \varphi$. Accounting for such fees, the portfolio $\pi$ has expected utility $\frac{1}{p} E_{P}^{y}\left[\left(X_{T}^{\pi} e^{-\varphi T}\right)^{p}\right]$. However, by the bound (14) the finite-horizon portfolio $\pi_{T}$ satisfies

$$
\frac{1}{p} E_{P}^{y}\left[\left(X_{T}^{\pi_{T}} e^{-\varphi_{T} T}\right)^{p}\right] \leq \frac{1}{p} E_{P}^{y}\left[\left(M_{T}^{\eta}\right)^{q}\right]^{1-p} e^{-p \varphi_{T} T} .
$$

Hence, $\pi$ is preferred to $\pi_{T}$ when

$$
\varphi_{T}-\varphi \geq \frac{1}{p}\left(\frac{1}{T} \log E_{P}^{y}\left[\left(M_{T}^{\eta}\right)^{q}\right]^{1-p}-\frac{1}{T} \log E_{P}^{y}\left[\left(X_{T}^{\pi}\right)^{p}\right]\right) .
$$

For a long-run optimal pair $(\pi, \eta)$, the limit of the right-hand side is zero. Thus, for any minimal difference in fees, a long-run optimal portfolio is preferable for investors with sufficiently long horizons.

2. Long-run analysis. The construction of long-run optimal portfolios $\pi$ and risk premia $\eta$ takes place in two steps. In the first step, Theorem $7 \mathrm{com}-$ putes the finite-horizon performance of the long-run optimal "candidates" $\pi$ and $\eta$. In the second step, Theorem 9 establishes a sufficient condition for long-run optimality, requiring that the bounds found in the first step converge at long horizons.

The candidate long-run optimal $\pi$ and $\eta$ crucially depend upon the solution of the quasi-linear partial differential equation (PDE) in (22), which acts as a long-run version of the Hamilton-Jacobi-Bellman equation. Thus, Theorems 7 and 9 are akin to verification theorems of stochastic control theory, but for the asymptotic objective in Definition 6. An advantage of these results is that they only rely on the local properties of the processes $(R, Y)$, avoiding the knowledge of the transition density of $Y$, which may be very complicated if known at all.

The second part of this section studies the existence of solutions to the ergodic Bellman equation in (22). Theorems 13, 18 and Proposition 15 below adapt the results of Kaise and Sheu (2006) to the present setting and under some extra conditions in addition to the assumptions of Theorems 7 and 9 . Their main message is that the quasi-linear PDE generally admits only one candidate for long-run optimality. As shown with examples in Section 4, this candidate may or may not be long-run optimal. 
2.1. Main results. Recall that, although their dependence on $y$ is omitted to alleviate notation, $b, \mu, \Sigma, \Upsilon, A, \phi$ and $v$ are functions of the state variable $y$.

Theorem 7. In addition to Assumptions 1 and 2, assume that:

(i) $v \in C^{2}(E, \mathbb{R})$ and $\lambda \in \mathbb{R}$ solve the ergodic HJB equation (cf. Section 3.2)

$$
\begin{aligned}
p r- & \frac{q}{2} \mu^{\prime} \Sigma^{-1} \mu+\frac{1}{2} \nabla v^{\prime}\left(A-q \Upsilon^{\prime} \Sigma^{-1} \Upsilon\right) \nabla v \\
& +\nabla v^{\prime}\left(b-q \Upsilon^{\prime} \Sigma^{-1} \mu\right)+\frac{1}{2} \operatorname{tr}\left(A D^{2} v\right)=\lambda ;
\end{aligned}
$$
for

(ii) there is a unique solution $\left(\hat{P}^{r, y}\right)_{r \in \mathbb{R}^{n}, y \in E}$ to the martingale problem

$$
\begin{gathered}
\hat{L}=\frac{1}{2} \sum_{i, j=1}^{n+k} \tilde{A}^{i, j}(x) \frac{\partial^{2}}{\partial x_{i} \partial x_{j}}+\sum_{i=1}^{n+k} \hat{b}^{i}(x) \frac{\partial}{\partial x_{i}}, \\
\hat{b}=\left(\begin{array}{c}
\frac{1}{1-p}(\mu+\Upsilon \nabla v) \\
b-q \Upsilon^{\prime} \Sigma^{-1} \mu+\left(A-q \Upsilon^{\prime} \Sigma^{-1} \Upsilon\right) \nabla v
\end{array}\right),
\end{gathered}
$$

where $\tilde{A}$ is as in (6).

Then, the pair $(\pi, \eta)$ given by

$$
\pi=\frac{1}{1-p} \Sigma^{-1}(\mu+\Upsilon \nabla v), \quad \eta=\nabla v
$$

satisfies the equalities

$$
\begin{aligned}
E_{P}^{y}\left[\left(X_{T}^{\pi}\right)^{p}\right] & =e^{\lambda T+v(y)} E_{\hat{P}}^{y}\left[e^{-v\left(Y_{T}\right)}\right], \\
E_{P}^{y}\left[\left(M_{T}^{\eta}\right)^{q}\right]^{1-p} & =e^{\lambda T+v(y)} E_{\hat{P}}^{y}\left[e^{-(1 /(1-p)) v\left(Y_{T}\right)}\right]^{1-p} .
\end{aligned}
$$

REMARK 8. Equations (25) and (26) provide lower and upper bounds on finite-horizon expected utility. Indeed, the duality inequality (14) yields

$$
\begin{aligned}
\frac{1}{p} e^{\lambda T+v(y)} E_{\hat{P}}^{y}\left[e^{-v\left(Y_{T}\right)}\right] & =\frac{1}{p} E_{P}^{y}\left[\left(X_{T}^{\pi}\right)^{p}\right] \leq u_{T}(y) \leq \frac{1}{p} E_{P}^{y}\left[\left(M_{T}^{\eta}\right)^{q}\right]^{1-p} \\
& =\frac{1}{p} e^{\lambda T+v(y)} E_{\hat{P}}^{y}\left[e^{-(1 /(1-p)) v\left(Y_{T}\right)}\right]^{1-p} .
\end{aligned}
$$

Combining (25) and (26) with (19) yields the central quantitative implication: an upper bound on the certainty equivalent loss

$$
l_{T} \leq \frac{1}{p}\left(\frac{1}{T} \log E_{\hat{P}}^{y}\left[e^{-(1 /(1-p)) v\left(Y_{T}\right)}\right]^{1-p}-\frac{1}{T} \log E_{\hat{P}}^{y}\left[e^{-v\left(Y_{T}\right)}\right]\right) .
$$


Theorem 7 now reduces the long-run optimality (Definition 6$)$ of $(\pi, \eta)$ to the condition that the right-hand side in (27) converges to zero. Theorem 9 below provides a criterion that covers most applications and Proposition 25 below shows a model in which this criterion is sharp, in that it holds for all the parameter values for which long-run optimality holds.

TheOREM 9. If, in addition to the assumptions of Theorem 7:

(i) the random variables $\left(Y_{t}\right)_{t \geq 0}$ are $\hat{P}^{y}$-tight ${ }^{9}$ in $E$ for each $y \in E$;

(ii) $\sup _{y \in E} F(y)<+\infty$, where $F \in C(E, \mathbb{R})$ is defined as

$$
F=\left\{\begin{array}{lc}
\left(p r-\lambda-\frac{q}{2} \mu^{\prime} \Sigma^{-1} \mu+\frac{q}{2} \nabla v^{\prime} \Upsilon^{\prime} \Sigma^{-1} \Upsilon \nabla v\right) e^{-v}, & p<0, \\
\left(p r-\lambda-\frac{q}{2} \mu^{\prime} \Sigma^{-1} \mu-\frac{q}{2} \nabla v^{\prime}\left(A-\Upsilon^{\prime} \Sigma^{-1} \Upsilon\right) \nabla v\right) e^{-(1 /(1-p)) v}, & 0<p<1 .
\end{array}\right.
$$

Then the pair $(\pi, \eta)$ in (24) is long-run optimal.

Section 4 shows how to check conditions (i) and (ii) in typical classes of models.

REMARK 10. A sufficient condition for (i) above to hold is that there exists a nonnegative $\psi \in C(E, \mathbb{R})$ such that, for each $n$, the level set $K_{n} \equiv$ $\{y \in E: \psi(y) \leq n\}$ is compact and that $M \equiv \sup _{t \geq 0} E_{\hat{P}}^{y}\left[\psi\left(Y_{t}\right)\right]<\infty$. If such a $\psi$ exists, then Markov's inequality implies that, for each $n$

$$
\sup _{t \geq 0} \hat{P}^{y}\left(Y_{t} \in K_{n}^{c}\right) \leq \frac{1}{n} \sup _{t \geq 0} E_{\hat{P}}^{y}\left[\psi\left(Y_{t}\right)\right]=\frac{M}{n} .
$$

Thus, $\hat{P}^{y}$ tightness in $E$ follows.

2.2. Solutions to the ergodic Bellman equation. This subsection provides conditions for the existence of a solution pair $(v, \lambda)$ to $(22)$, such that the tightness condition in Theorem 9 holds. These results are obtained adapting the arguments in Kaise and Sheu (2006) to the present setting. Define $\Lambda$ as the set of $\lambda \in \mathbb{R}$ for which a solution $v$ to $(22)$ exists,

$$
\Lambda=\left\{\lambda \in \mathbb{R} \mid \exists v \in C^{2, \gamma}(E, \mathbb{R}) \text { solving }(22)\right\} .
$$

$\Lambda$ depends both on the region $E$ and on the coefficients in the PDE (22). The foregoing results require the following assumption on the region $E$, which holds in virtually all models in the literature.

\footnotetext{
${ }^{9}$ Recall that a family of $E$-valued random variables $\left(X_{t}\right)_{t \geq 0}$ is $P$-tight in $E$ if the induced measures $\left(P \circ X_{t}^{-1}\right)_{t \geq 0}$ form a tight family in $M_{1}(E)$, the space of Borel measures on $E$. Thus, $\left(X_{t}\right)_{t \geq 0}$ is $P$-tight in $E$ if for each $\varepsilon>0$ there exists a compact $K_{\varepsilon} \subset E$ such that $\sup _{t \geq 0} P\left(X_{t} \in K_{\varepsilon}^{c}\right) \leq \varepsilon$.
} 
Assumption 11. There exist $y_{0} \in E$ and a sequence of bounded open subsets $E_{n} \subset E$, star-shaped ${ }^{10}$ with respect to $y_{0}$ and with a $C^{2, \gamma}$ boundary, and strictly increasing to $E$, in that $E=\bigcup_{n=1}^{\infty} E_{n}$ and $\bar{E}_{n} \cap\left(E \backslash E_{n+1}\right)=\varnothing$.

This assumption is satisfied by any convex set $E$ for which there is a convex function $\psi \in C^{2, \gamma}(E, \mathbb{R})$ such that $\psi(y) \uparrow \infty$ as $y \rightarrow \partial E$. In this case, it suffices to set $E_{n}=\{y \in E: \psi(y)<n\}$. The next assumption requires that the potential $p r-\frac{q}{2} \mu^{\prime} \Sigma^{-1} \mu$ is bounded from above. When it does not hold, solutions to (22) may not exist [Pinsky (1995), Chapter 4.5].

ASsumption 12. $\sup _{y \in E}\left(\operatorname{pr}(y)-\frac{q}{2} \mu(y)^{\prime} \Sigma(y)^{-1} \mu(y)\right)<\infty$.

Note that Assumption 12 always holds if $p<0$, and the interest rate $r$ is bounded from below, which is a typical situation in financial models. With Assumptions 1, 2 and 11, denote by $(R, Y)$ the coordinate process of the solution $\left(P^{y}\right)_{y \in E}$ of the martingale problem corresponding to the operator $L$ from (6). Regarding the state variable $Y$, the statement of existence results requires a few basic definitions in ergodic theory [see Pinsky (1995), Pinchover (1992) for more details]. $Y$ is transient if $P^{y}(Y$ is eventually in $E \backslash E_{n}$ for all $\left.n \geq N(\omega)\right)=1$ for all $n \geq 1$ and $y \in E$. $Y$ is recurrent if $P^{x}(\tau(\varepsilon, y)<\infty)=1$ for all $x, y \in E$ and $\varepsilon>0$, where $\tau(\varepsilon, y)=\inf _{\sim}\{t \geq$ 0||$\left.Y_{t}-y \mid \leq \varepsilon\right\}$. If $Y$ is recurrent, there exists some $\tilde{\phi}>0$ such that $\tilde{L} \tilde{\phi}=0$, where $\tilde{L}$ is the formal adjoint to $L . Y$ is positive recurrent, or ergodic, if $\int_{E} \tilde{\phi} d y<\infty$, and null recurrent otherwise. If $Y$ is positive recurrent and $\tilde{\phi}$ is normalized to be a probability density, then for all $y \in E$ and $f \in L^{1}(E, \tilde{\phi})$

$$
\lim _{T \uparrow \infty} E_{P}^{y}\left[f\left(Y_{T}\right)\right]=\int_{E} f \tilde{\phi} d x .
$$

If $Y$ is ergodic, then (30) implies that $Y$ is $P^{y}$-tight in $E$ for each $y \in E$, and for all $y \in E$ and $\left(t_{n}\right)_{n \geq 1} \uparrow \infty$, the measures $\left(P^{y} \circ Y_{t_{n}}\right)^{-1}$ weakly converge to the measure with density $\tilde{\phi}$, which does not depend upon the starting point $y \in E$. With these definitions and results, the following theorem shows that there exists only one possible pair $(\lambda, v)$, solving (22), which can lead to long-run optimality.

THEOREM 13. Let Assumptions 1, 11 and 12 hold. Then there exists $\lambda_{c} \in \mathbb{R}$ such that $\Lambda=\left[\lambda_{c}, \infty\right)$. Furthermore, $Y$ is $\left(\hat{P}^{y}\right)_{y \in E}$-transient for any $\hat{P}$ corresponding to a solution $(v, \lambda)$ of (22) with $\lambda>\lambda_{c}$.

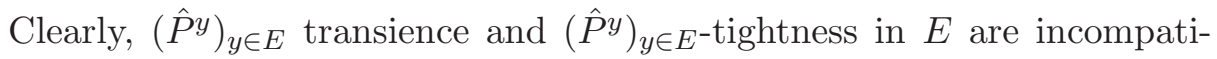
ble with one another. Furthermore, since ergodicity implies tightness, the

\footnotetext{
${ }^{10}$ Recall that $F \subseteq \mathbb{R}^{k}$ is star-shaped for some $x_{0} \in F$ if for each $x \in F$ the segment $\left\{\alpha x_{0}+(1-\alpha) x ; 0 \leq \alpha \leq 1\right\}$ is contained within $F$. A convex set is star-shaped with respect to any of its points.
} 
question is whether the pair $\left(\lambda_{c}, v_{c}\right)$ makes $Y$ ergodic under $\left(\hat{P}^{y}\right)_{y \in E}$. The following results give conditions under which this is indeed the case. The first proposition is valid for a single state variable and constant correlations $\rho$. In this case, (22) linearizes under a power transformation, and classical tests for transience and recurrence of one-dimensional diffusions apply. The second proposition considers the general multidimensional case but under a stronger restriction on the potential term.

2.2.1. One state, constant correlations. An important case leads to substantial simplifications.

Assumption 14. Let Assumptions 1 and 12 hold. Further, assume that:

(i) $E=(\alpha, \beta)$ with $-\infty \leq \alpha<\beta \leq \infty$.

(ii) $\rho^{\prime} \rho=\Upsilon^{\prime} \Sigma^{-1} \Upsilon / A$ is constant.

Note that Assumption 11 is always satisfied for a single state variable. Set

$$
\delta=\frac{1}{1-q \rho^{\prime} \rho} .
$$

The change of variable $\phi=\exp (v / \delta)$, essentially equivalent to the power transformation of Zariphopoulou (2001), reduces the quasi-linear ODE in (22) to the linear ODE

$$
\frac{A}{2} \ddot{\phi}+\left(b-q \Upsilon^{\prime} \Sigma^{-1} \mu\right) \dot{\phi}+\frac{1}{\delta}(V-\lambda) \phi=0 .
$$

Let $\lambda \in \Lambda$ and let $\phi \in C^{2, \gamma}(E, \mathbb{R})$ with $\phi>0$ be a solution to (32) obtained by $\phi=\exp (v / \delta)$. Under $\hat{P}^{y}, Y$ has the dynamics

$$
d Y_{t}=\left(b-q \Upsilon^{\prime} \Sigma^{-1} \mu+A \frac{\dot{\phi}}{\phi}\right) d t+a d \hat{W}_{t} .
$$

Using Feller's test for explosions, the following proposition [Pinsky (1995), Corollary 5.1.11] gives sufficient conditions for $Y$ to be $\hat{P}^{y}$-tight in $E$ for the candidate optimal pair $\left(\lambda_{c}, \phi_{c}\right)$.

Proposition 15. Let Assumption 14 hold, let $\left(\lambda_{c}, v_{c}\right)$ be as in Theorem 13 and let $\phi_{c}=\exp \left(v_{c} / \delta\right)$. Denote by

$$
m_{\nu}(y)=\frac{1}{A(y)} \exp \left(\int_{y_{0}}^{y} \frac{2\left(b-q \Upsilon^{\prime} \Sigma^{-1} \mu\right)(z)}{A(z)} d z\right)
$$

where $y_{0} \in(\alpha, \beta)$. Then, the family of random variables $\left(Y_{t}\right)_{t \geq 0}$ is $\hat{P}^{y}$-tight in $E$ if and only if

$$
\int_{\alpha}^{y_{0}} \frac{1}{\phi_{c}^{2} A m_{\nu}} d y=\int_{y_{0}}^{\beta} \frac{1}{\phi_{c}^{2} A m_{\nu}} d y=\infty \quad \text { and } \quad \int_{\alpha}^{\beta} \phi_{c}^{2} m_{\nu} d y<\infty .
$$


2.2.2. The general case. This subsection treats the general case of $k$ state variables under the following assumption.

Assumption 16. There exists a function $w \in C^{2, \gamma}(E, \mathbb{R})$ such that

$$
\begin{aligned}
\lim _{n \uparrow \infty} \sup _{E \backslash E_{n}}\left(p r-\frac{q}{2}\right. & \mu^{\prime} \Sigma^{-1} \mu+\frac{1}{2} \nabla w^{\prime}\left(A-q \Upsilon^{\prime} \Sigma^{-1} \Upsilon\right) \nabla w \\
& \left.+\nabla w^{\prime}\left(b-q \Upsilon^{\prime} \Sigma^{-1} \mu\right)+\frac{1}{2} \operatorname{tr}\left(A D^{2} w\right)\right)=-\infty
\end{aligned}
$$

REMARK 17. For general regions $E$, condition (36) plays a similar role as condition (A3) in Kaise and Sheu (2006). Assumption 16 is satisfied, for example, when

$$
\lim _{n \uparrow \infty} \sup _{E \backslash E_{n}}\left(p r-\frac{q}{2} \mu^{\prime} \Sigma^{-1} \mu\right)=-\infty
$$

In this case, $w \equiv 1$ satisfies (36).

In this setting, the main existence criterion is the following.

TheOREM 18. Let Assumptions 1, 11, 12 and 16 hold and let $\left(\lambda_{c}, v_{c}\right)$ be as in Theorem 13. Then $v_{c}$ is unique up to an additive constant and $\left(Y_{t}\right)_{t \geq 0}$ is $\hat{P}^{y}$-tight in $E$ for all $y \in E$.

Proposition 25 in Section 4 below shows that long-run optimality may still fail, even when the tightness condition is satisfied for the pair $\left(\lambda_{c}, v_{c}\right)$. The reason is that, even if $Y$ is ergodic under $\left(\hat{P}^{y}\right)_{y \in E}$ with invariant density $\tilde{\phi}$, the ergodic property in (30) may not hold for (25) and (26) because the functions

$$
\exp (-v(y)), \quad \exp \left(-(1-p)^{-1} v(y)\right)
$$

therein may not be in $L^{1}(E, \tilde{\phi})$. Thus, long-run optimality requires additional assumptions, such as (28) in Theorem 9.

If the functions in $(38)$ are in $L^{1}(E, \tilde{\phi})$, then (30) yields additional information about the speed at which the certainty equivalent loss $l_{T}$ converges to zero in the limit of a long horizon. The following proposition provides such a result, in the case of a single state variable. Recall that risk aversion is $\gamma=1-p=1 /(1-q)$. The main message is that long-run optimality may fail only for (i) high risk aversion and highly incomplete market or (ii) low risk aversion and nearly complete market. In particular, for $-1<q \leq 1 / 2$ (i.e., risk aversion within $1 / 2$ and 2), long-run optimality holds for any level of 
incompleteness. In addition, for $q>1 / 2$ (resp., $q<-1$ ), long-run optimality holds if $\rho^{\prime} \rho<1 / 2$ (resp., $\rho^{\prime} \rho>1 / 2$ ).

Section 4 takes up this issue in specific models, obtaining necessary and sufficient conditions. By contrast, the following sufficient condition holds under general assumptions, regardless of the model considered.

Proposition 19. Let Assumption 14 hold, and assume that $m_{\nu}$ in (34) satisfies $\int_{E} m_{\nu} d y<\infty$. If $\left(\lambda_{c}, v_{c}\right)$ are such that $Y$ is $\left(\hat{P}^{y}\right)_{y \in E \text {-ergodic, then }}$ long-run optimality holds if

$$
\rho^{\prime} \rho \in \begin{cases}{\left[0, \frac{1}{2 q}\right],} & \text { for } \frac{1}{2}<q<1, \\ {[0,1],} & \text { for }-1 \leq q \leq \frac{1}{2}, q \neq 0, \\ {\left[\frac{1+q}{2 q}, 1\right],} & \text { for } q<-1 .\end{cases}
$$

In such cases, there exists a constant $K>0$ such that the certainty equivalent loss $l_{T}$ satisfies

$$
0 \leq \limsup _{T \uparrow \infty} T l_{T} \leq K
$$

The next corollary states in an important special case, which does not even require the knowledge of the principal eigenfunction $\phi$, since $m_{\nu}$ only depends on the model parameters.

Corollary 20. Under Assumption 14, if $\int_{E} m_{\nu} d y<\infty$ and (37) are satisfied, then long-run optimality holds for $q$ and $\rho^{\prime} \rho$ satisfying (39).

\section{Implications and ramifications.}

3.1. The myopic probability. The bounds (25) and (26) in Theorem 7 and assumption (i) in Theorem 9 depend on the equivalent probability $\hat{P}^{y}$, which plays a pivotal role in long-run analysis. In general, $\hat{P}^{y}$ is neither the physical probability $P^{y}$ nor a risk-neutral probability. Instead, its interpretation becomes clear from its dynamics, which is (for $\hat{P}$-Brownian motions $\hat{Z}, \hat{W}$ )

$$
\begin{aligned}
& d R_{t}=\frac{1}{1-p}(\mu+\Upsilon \nabla v) d t+\sigma d \hat{Z}_{t} \\
& d Y_{t}=\left(b-q \Upsilon^{\prime} \Sigma^{-1} \mu+\left(A-q \Upsilon^{\prime} \Sigma^{-1} \Upsilon\right) \nabla v\right) d t+a d \hat{W}_{t} .
\end{aligned}
$$

Compare the original model, with price dynamics under $P^{y}$ and power utility $x^{p} / p$, to the auxiliary model under $\hat{P}^{y}$ with logarithmic utility. The long-run optimal portfolio in the two models coincide. The first one is sim- 
ply in (24), while the second one follows from the usual formula $\pi=\Sigma^{-1} \hat{\mu}$, where $\hat{\mu}=\frac{1}{1-p}(\mu+\Upsilon \nabla v)$ are the expected returns under $\hat{P}^{y}$. Thus a long horizon, power-utility investor under the probability $P^{y}$ behaves exactly as a myopic (or logarithmic) investor under $\hat{P}^{y}$.

This observation shows that $\hat{P}^{y}$ corresponds to the long horizon limit of the probability $\mathbf{R}$ considered by Kramkov and Sîrbu (2006a, 2006b, 2007) in finite horizon, in the context of sensitivity analysis pricing of option prices. Černý and Kallsen (2007) study mean-variance hedging for semimartingales and obtain optimal strategies in terms of the predictable characteristics of asset prices under an opportunity neutral probability $P^{*}$, which is similar in spirit to $\hat{P}^{y}$, in that it reduces the mean-variance objective to a logarithmic utility objective.

3.2. Connections with stochastic control. Since the work of Merton (1969), most of the dynamic portfolio choice literature has employed stochastic optimal control as its main analytical tool. The relation between Theorems 7,9 and the stochastic control approach becomes clear by comparing equation (22) to the Hamilton-Jacobi-Bellman (HJB) equations of the utility maximization problem (10). Its value function $u(x, y, t)$ depends on the current wealth $x$, the current state $y$ and time $t$. The homogeneity of power utility entails that $u(x, y, t)=x^{p} e^{w(y, t)} / p$, thereby removing wealth from the reduced value function $w$. The corresponding HJB equation becomes [see, e.g., Pham (2002)]

$$
\begin{aligned}
-\frac{\partial w}{\partial t}= & p r-\frac{q}{2} \mu^{\prime} \Sigma^{-1} \mu+\frac{1}{2} \nabla w^{\prime}\left(A-q \Upsilon^{\prime} \Sigma^{-1} \Upsilon\right) \nabla w \\
& +\nabla w^{\prime}\left(b-q \Upsilon^{\prime} \Sigma^{-1} \mu\right)+\frac{1}{2} \operatorname{tr}\left(A D^{2} w\right)
\end{aligned}
$$

with the terminal condition $w(y, T)=0$. Instead, the main PDE (22) is

$$
\begin{aligned}
\lambda= & p r-\frac{q}{2} \mu^{\prime} \Sigma^{-1} \mu+\frac{1}{2} \nabla v^{\prime}\left(A-q \Upsilon^{\prime} \Sigma^{-1} \Upsilon\right) \nabla v \\
& +\nabla v^{\prime}\left(b-q \Upsilon^{\prime} \Sigma^{-1} \mu\right)+\frac{1}{2} \operatorname{tr}\left(A D^{2} v\right) .
\end{aligned}
$$

In the former equation, the unknown function $w$ depends on both time $t$ and the state $y$, while $v$ in the latter equation only depends on the state, although the constant $\lambda$ is also unknown. Indeed, the former equation reduces to the latter under the restriction

$$
w(t, y)=\lambda(T-t)+v(y) .
$$

This restriction gains analytical tractability by reducing the dimension of the problem. The price of the tractability gain is that solutions of the timehomogeneous equation in general do not satisfy the boundary condition 
and, therefore, are not exactly optimal at any time-horizon (except in the trivial case $v=0$, arising with logarithmic utility or constant investment opportunities).

A special case of equation (22) appears in the risk-sensitive control approach to optimal investment initiated by Bielecki and Pliska (1999). In a linear diffusion model, they study the problem

$$
\max _{\pi} \liminf _{T \rightarrow \infty} \frac{1}{T} \log E\left[\left(X_{T}^{\pi}\right)^{p}\right],
$$

where the supremum is taken over all progressively measurable strategies. Risk-sensitive control relies on control techniques to establish the existence and uniqueness to the homogeneous equation, then attempts to establish its optimality in the sense of (42). Fleming and Sheu (2000, 2002) carry out this program under the assumption that $|p|$ is small, that is, if risk aversion is close enough to the logarithmic case. The results in this paper, which apply to general nonlinear models, shed new light on this literature by characterizing finite-horizon performance. For example, Proposition 25 below relaxes the restriction of $|p|$ small to a necessary and sufficient condition and explains the economic intuition behind it.

3.3. Long-run q-optimal measure. For each value of the risk-aversion parameter $1-p$, the risk premia $\eta$ in (24) deliver a pricing rule for derivatives involving the partially unhedgeable state variable $Y$. The martingale measure $Q^{\eta}$ corresponding to the risk premia $\eta$ is a long-run version of the minimax martingale measure of He and Pearson (1991), called q-optimal measure by Hobson (2004) and Henderson (2005). Its formal dynamics is

$$
\left\{\begin{array}{l}
d R_{t}=\sigma d \tilde{Z}_{t} \\
d Y_{t}=\left(b-\Upsilon^{\prime} \Sigma^{-1} \mu+\left(A-\Upsilon^{\prime} \Sigma^{-1} \Upsilon\right) \nabla v\right) d t+a d \tilde{W}_{t}
\end{array}\right.
$$

for some Brownian motions $\tilde{Z}$ and $\tilde{W}$. Since this dynamics is distinct from the one under $P^{y}$ and $\hat{P}^{y}$, in general it is necessary to check its wellposedness, in the form of Assumption 2.

Observe that the drift of $Y$ under the $q$-optimal measure has three components. The first term $b$ is the drift under the original measure $P^{y}$. The second term $\Upsilon^{\prime} \Sigma^{-1} \mu$ is the risk-neutral adjustment due to the correlation between the returns and the state shocks. The last term $\left(A-\Upsilon^{\prime} \Sigma^{-1} \Upsilon\right) \nabla v$ accounts for preferences which enter the equation through $v$.

3.4. Complete and fully incomplete as duals. The formulas in (24) highlight the symmetric aspects of complete markets, where $A=\Upsilon^{\prime} \Sigma^{-1} \Upsilon$ identically and fully incomplete markets, where $\Upsilon=0$. In a complete market the 
pricing problem is trivial, as the dynamics in (43) becomes independent of the preference parameter $p$,

$$
\left\{\begin{array}{l}
d R_{t}=\sigma d \tilde{Z}_{t} \\
d Y_{t}=\left(b-\Upsilon^{\prime} \Sigma^{-1} \mu\right) d t+a d \tilde{W}_{t}
\end{array}\right.
$$

However, the investment problem is nontrivial because the optimal portfolio includes a component that perfectly hedges the state variables.

Conversely, in a fully incomplete market, myopic portfolios are optimal because all portfolios evolve orthogonally to state variables. However, a latent hedging motive remains present and generates nonzero risk premia for state variables, which have a potential as hedging instruments. In both cases, it is market dynamics, and not preferences, which make either the investment or the pricing problem trivial. By contrast, logarithmic preferences or constant $\mu$ and $\sigma$ remove the intertemporal hedging motive entirely, making both problems trivial.

The quasi-linear ODE in (22) becomes linear under a transformation in both the complete and fully incomplete cases, as in the one state variable case discussed in Section 2.2.1. In the complete case, the transformation $\phi=e^{(1-q) v}$ leads to the linear equation

$$
\frac{1}{2} \operatorname{tr}\left(A D^{2} \phi\right)+\nabla \phi^{\prime}\left(b-q \Upsilon^{\prime} \Sigma^{-1} \mu\right)+(1-q)\left(p r-\frac{q}{2} \mu^{\prime} \Sigma^{-1} \mu-\lambda\right) \phi=0 .
$$

In the fully incomplete case, the transformation $\phi=e^{v}$ leads to the linear equation

$$
\frac{1}{2} \operatorname{tr}\left(A D^{2} \phi\right)+\nabla \phi^{\prime} b+\left(p r-\frac{q}{2} \mu^{\prime} \Sigma^{-1} \mu-\lambda\right) \phi=0 .
$$

The criticality theory of Pinsky (1995) applies to these cases under multivariate restrictions similar to those given in Assumption 14, with $\delta=\frac{1}{1-q}$ in the complete case and $\delta=1$ in the fully incomplete case. Furthermore, the multivariate results of Theorem 18 apply as long as (36) can be verified.

3.5. Long-run decomposition. The bounds (25) and (26) decompose expected utility and its dual into a common "long-run" component $e^{\lambda T}$, and two "transient" components, in a close analogy to Hansen and Scheinkman (2009). For a multiplicative functional $N$ of a Markov process $Y$, they propose the decomposition

$$
N_{t}=\exp (\rho t) \frac{\varphi(y)}{\varphi\left(Y_{t}\right)} \hat{N}_{t}
$$

where $\rho$ and $\varphi$ are, respectively, the principal eigenvalue and eigenfunction of the infinitesimal generator of $Y$ and $\hat{N}_{t}$ is a martingale. The bounds (25) 
and (26) yield similar expressions for terminal utilities and their dual counterparts

$$
\left(X_{T}^{\pi}\right)^{p}=e^{\lambda T} \frac{e^{v(y)}}{e^{v\left(Y_{T}\right)}} \frac{d \hat{P}^{y}}{d P^{y}} \quad\left(M_{T}^{\eta}\right)^{q}=\left(e^{\lambda T} \frac{e^{v(y)}}{e^{v\left(Y_{T}\right)}}\right)^{1 /(1-p)} \frac{d \hat{P}^{y}}{d P^{y}} .
$$

These decompositions are precisely of the form in (47), with the difference that on the dual side the transient components are powers of $e^{v}$, as opposed to $e^{v}$ itself. Note also that the operator in (22) is not the generator of $Y$ under either $P^{y}$ or $\hat{P}^{y}$, since it is nonlinear, has nonzero potential and has a different drift. Further, the interpretation of $e^{\lambda T}$ as a long-run component hinges on the condition that the $\hat{P}^{y}$-expectation of transient components has a less than exponential growth, which means that long-run optimality holds. This is not always the case; the examples in Section 4 show how parameter restrictions are necessary even in the most common models.

3.6. Large deviations. Theorems 7 and 9 are closely related to the results of Donsker and Varadhan $(1975,1976,1983)$ on large deviations of occupation times for diffusions. Though the results also hold in the multidimensional case of $k>1$ state variables, the following discussion considers a single state variable for simplicity of notation.

Let $E=(\alpha, \beta)$ for $-\infty \leq \alpha<\beta \leq \infty$ and consider a diffusion $Y$ with generator $L^{Y}$ from (7) (with $k=1$ ), assuming that the coefficients $A$ and $b$ are such that $Y$ is positive recurrent under $\left(P^{y}\right)_{y \in E}$. Let $m$ be the invariant measure which has a density by (30). With a slight abuse of notation, let $m(d y)=m(y) d y$.

Denote by $M_{1}(\alpha, \beta)$ the space of Borel probability measures on $(\alpha, \beta)$. Under certain conditions on $Y$, Donsker and Varadhan show that, for all continuous bounded functions $V:(\alpha, \beta) \mapsto \mathbb{R}$ and all $y \in(\alpha, \beta)$,

$$
\lim _{T \rightarrow \infty} \frac{1}{T} \log E_{P}^{y}\left[\exp \left(\int_{0}^{T} V\left(Y_{t}\right) d t\right)\right]=\sup _{\mu \in M_{1}((\alpha, \beta))}\left(\int_{\alpha}^{\beta} V d \mu-I(\mu)\right) .
$$

For $\mu \in M_{1}(\alpha, \beta)$ absolutely continuous with respect to $m$ (and hence, the Lebesgue measure) and with density $\mu(y)$ such that $\psi(y)^{2} \equiv \mu(y) / m(y)$ satisfies certain regularity and decay conditions, the rate function $I(\mu)$ reduces to

$$
I(\mu)=\frac{1}{2} \int_{\alpha}^{\beta} A(\dot{\psi})^{2} m d y .
$$

Using this representation for the rate function, the following heuristic argument shows the relation between the Donsker and Varadhan (1975) theory and long-run optimality. Consider the terminal utility of a portfolio $\pi\left(Y_{t}\right)$ for some function $\pi: E \mapsto \mathbb{R}^{n}$,

$$
\left(X_{T}^{\pi}\right)^{p}=\exp \left(\int_{0}^{T}\left(p r+p \pi^{\prime} \mu+\frac{1}{2} p(p-1) \pi^{\prime} \Sigma \pi\right) d t\right) \mathcal{E}\left(\int_{0}^{.} p \pi^{\prime} \sigma d Z_{t}\right)_{T} .
$$


Define $P_{\pi}^{y}$ by setting $\frac{d P_{\pi}^{y}}{d P^{y}}$ equal to the stochastic exponential in the last term of this equation. It follows that

$$
E_{P}^{y}\left[\left(X_{T}^{\pi}\right)^{p}\right]=E_{P_{\pi}}^{y}\left[\exp \left(\int_{0}^{T}\left(p r+p \pi^{\prime} \mu+\frac{1}{2} p(p-1) \pi^{\prime} \Sigma \pi\right) d t\right)\right] .
$$

Assuming they may be applied under $P_{\pi}^{y}$, the Donsker and Varadhan asymptotics (48) yield

$$
\begin{aligned}
\lim _{T \rightarrow \infty} & \frac{1}{T} \log E_{P}^{y}\left[\left(X_{T}^{\pi}\right)^{p}\right] \\
=\sup _{\psi \in L_{1}^{2}\left(m_{\pi}\right)} \int_{\alpha}^{\beta}\left(\left(p r+p \pi^{\prime} \mu+\frac{1}{2} p(p-1) \pi^{\prime} \Sigma \pi\right) \psi^{2}\right. & \\
& \left.-\frac{1}{2} A(\dot{\psi})^{2}\right) m_{\pi} d y,
\end{aligned}
$$

where $m_{\pi}$ is the invariant density of $Y$ under $P_{\pi}^{y}$ and $L_{1}^{2}\left(m_{\pi}\right)$ is the unit disc in $L^{2}\left(m_{\pi}\right)$. In a similar manner to (34) in Proposition $15, m_{\pi}$ admits the formula

$$
m_{\pi}(y)=\frac{1}{A(y)} \exp \left(\int_{y_{0}}^{y} \frac{2\left(b+p \Upsilon^{\prime} \pi\right)(z)}{A(z)} d z\right) .
$$

Here, $y_{0}$ is some interior point in $E$. To make the dependence on the portfolio $\pi$ explicit, the change of variable $\psi^{2} m_{\pi}=\phi^{2} m_{\nu}$ yields $\left[m_{\nu}\right.$ is defined in $(34)$ ]

$$
\frac{\dot{\psi}}{\psi}=\frac{\dot{\phi}}{\phi}+\frac{\dot{m}_{\nu}}{2 m_{\nu}}-\frac{\dot{m}_{\pi}}{2 m_{\pi}}=\frac{\dot{\phi}}{\phi}-A^{-1}\left(q \Upsilon^{\prime} \Sigma^{-1} \mu+p \Upsilon^{\prime} \pi\right) .
$$

Substituting (50) into (49), the utility growth rate becomes

$$
\sup _{\phi \in L_{1}^{2}\left(m_{\nu}\right)} \int_{\alpha}^{\beta}\left(\pi^{\prime} \mathbf{A} \pi+\pi^{\prime} \mathbf{B}+\mathbf{C}\right) \phi^{2} m_{\nu} d y,
$$

where

$$
\begin{aligned}
& \mathbf{A}=\frac{1}{2} p(p-1)\left(\Sigma-q \Upsilon A^{-1} \Upsilon^{\prime}\right), \quad \mathbf{B}=p\left(\left(1_{n}-q \Upsilon A^{-1} \Upsilon^{\prime} \Sigma^{-1}\right) \mu+\Upsilon \frac{\dot{\phi}}{\phi}\right), \\
& \mathbf{C}=p r-\frac{1}{2} A\left(\frac{\dot{\phi}}{\phi}\right)^{2}+q \mu^{\prime} \Sigma^{-1} \Upsilon \frac{\dot{\phi}}{\phi}-\frac{1}{2} q^{2} \mu^{\prime} \Sigma^{-1} \Upsilon A^{-1} \Upsilon^{\prime} \Sigma^{-1} \mu .
\end{aligned}
$$

The integrand is a quadratic function of $\pi$ and achieves its optimum at

$$
\pi=\frac{1}{1-p} \Sigma^{-1}\left(\mu+\delta \Upsilon \frac{\dot{\phi}}{\phi}\right)
$$


Thus, substituting (52) into (51), the utility growth rate reduces to

$$
\sup _{\phi \in L_{1}^{2}\left(m_{\nu}\right)} \int_{\alpha}^{\beta}\left(\left(p r-\frac{q}{2} \mu^{\prime} \Sigma^{-1} \mu\right) \phi^{2}-\frac{\delta}{2} A(\dot{\phi})^{2}\right) m_{\nu} d y .
$$

A similar reasoning on stochastic discount factors delivers the candidate long-run risk premia. The Euler-Lagrange equation associated to (53) is the ODE in (32). Thus, large deviations arguments act as a guide for producing the candidate long-run optimal policies.

This argument, which explains the formal connection with large deviations, is suggestive but only heuristic. The main reason is that the DonskerVaradhan asymptotics are correct under some delicate conditions which may fail to hold even in the simplest models.

4. Applications. This section applies the main results to two models, assuming that the investor is more risk averse than the log investor $(p<0)$. In the first model, the state variables follow a multivariate Ornstein-Uhlenbeck process that drives the drift of the return process. Under general conditions, this model admits a unique solution $v$ to (22), leading to $\hat{P}^{y}$ tightness. For a single state variable, long-run optimality is characterized in terms of precise parameter restrictions.

In the second model, interest rates, drifts and volatilities are stochastic. Each of these quantities is affine in a single common state variable, which follows a Feller diffusion. Although this model does not belong to the affine class, the long-run optimal portfolios and risk premia have very simple expressions.

The parametric restrictions required by long-run optimality lead under each single variate model to the same economic interpretation. Long-run optimality does not hold at the conjunction of three extreme situations: (i) high covariation between risk premia and state variables, (ii) nearly complete markets and (iii) high risk-aversion. To understand this phenomenon, recall that long-run optimality means that a time-homogenous strategy is approximately optimal on a long time interval. Thus, the sub-optimality of the long-run strategy in the latest part of the interval must lead to a small utility loss. Since the myopic component of the optimal finite-horizon portfolio is time-homogenous, any loss in utility is attributed to the intertemporal hedging component. All of the three extreme situations mentioned above concur to amplify the intertemporal hedging component. First, the covariation of risk premia is proportional to the hedging portfolios $\Sigma^{-1} \Upsilon$. Second, intertemporal hedging is more attractive in a nearly complete market, where state variables are almost replicable. Third, intertemporal hedging is higher for more risk-averse investors who reduce long-term risk at the expense of short-term return. 
4.1. Linear diffusion. This is the most common multivariate model, with constant covariance matrices $\Sigma, \Upsilon, A$ and drifts $r, \mu, b$ that are affine functions of the state variable. The dynamics is

$$
\left\{\begin{array}{l}
d R_{t}=\left(\mu_{0}+\mu_{1} Y_{t}\right) d t+\sigma d Z_{t} \\
d Y_{t}=-b Y_{t} d t+a d W_{t} \\
d\langle R, Y\rangle_{t}=\rho d t \\
r\left(Y_{t}\right)=r_{0}+r_{1}^{\prime} Y_{t}
\end{array}\right.
$$

where $\mu_{0} \in \mathbb{R}^{n}, \mu_{1} \in \mathbb{R}^{n \times k}, \sigma \in \mathbb{R}^{n \times n}, b \in \mathbb{R}^{k \times k}, a \in \mathbb{R}^{k \times k}, \rho \in \mathbb{R}^{n \times k}, r_{0} \in \mathbb{R}$ and $r_{1} \in \mathbb{R}^{k}$. Under this model, $E=\mathbb{R}^{k}$ and state variables follow a multivariate Ornstein-Uhlenbeck process. This setting is considered in most of the literature in risk sensitive control mentioned in the Introduction and it is also implicit in the use of vector autoregressions in the econometrics literature. The coefficients in (54) satisfy the following.

Assumption 21. All four matrices $\Sigma=\sigma \sigma^{\prime}, \mu_{1}^{\prime} \mu_{1}, b+b^{\prime}$ and $a$ are positive definite.

Assumption 21 implies that condition (37) holds for $p<0$. Hence, by Theorem 18 [or by the results in Kaise and Sheu (2006)], there is a unique pair $(\lambda, v)$ solving $(22)$ such that for each $y \in E,\left(Y_{t}\right)_{t>0}$ is $\hat{P}^{y}$-tight in $E$. The next theorem shows that $v$ is in fact quadratic.

Theorem 22. Let Assumption 21 hold for the model in (54). For $p<0$, equation (22) admits a unique solution $v$ such that for each $y \in \mathbb{R}^{k},\left(Y_{t}\right)_{t \geq 0}$ is $\hat{P}^{y}$-tight in $\mathbb{R}^{k}$. The solution is of the form $v(y)=v_{0}^{\prime} y-\frac{1}{2} y^{\prime} v_{1} y$, where the symmetric matrix $v_{1} \in \mathbb{R}^{k \times k}$ and the vector $v_{0} \in \mathbb{R}^{k}$ and satisfy the algebraic equations

$$
\begin{aligned}
& \left(v_{1}\left(A-q \Upsilon^{\prime} \Sigma^{-1} \Upsilon\right)+\left(b+q \Upsilon^{\prime} \Sigma^{-1} \mu_{1}\right)^{\prime}\right) v_{0}-p r_{1} \\
& \quad+q\left(\mu_{1}^{\prime}-v_{1} \Upsilon^{\prime}\right) \Sigma^{-1} \mu_{0}=0, \\
& v_{1}\left(A-q \Upsilon^{\prime} \Sigma^{-1} \Upsilon\right) v_{1}+v_{1}\left(b+q \Upsilon^{\prime} \Sigma^{-1} \mu_{1}\right)+\left(b+q \Upsilon^{\prime} \Sigma^{-1} \mu_{1}\right)^{\prime} v_{1} \\
& \quad-q \mu_{1}^{\prime} \Sigma^{-1} \mu_{1}=0 .
\end{aligned}
$$

The corresponding utility growth rate equals

$$
\begin{aligned}
\lambda= & p r_{0}-\frac{q}{2} \mu_{0}^{\prime} \Sigma^{-1} \mu_{0}+\frac{1}{2} v_{0}^{\prime}\left(A-q \Upsilon^{\prime} \Sigma^{-1} \Upsilon\right) v_{0} \\
& -q v_{0}^{\prime} \Upsilon^{\prime} \Sigma^{-1} \mu_{0}-\frac{1}{2} \operatorname{tr}\left(A v_{1}\right) .
\end{aligned}
$$

Equation (56) is a quadratic equation in the unknown matrix $v_{1}$ and it belongs to the class of matrix Riccati equations which arise in filtering theory and dynamical systems. It does not admit a closed-form solution in terms 
of matrix operations but numerical techniques for obtaining the solution are available [see Abou-Kandil et al. (2003), Chapter 2]. Once the matrix $v_{1}$ is known, the linear equation (55) yields a unique solution for $v_{0}$ and $\lambda$ is quadratic in $v_{0}$ and linear in $v_{1}$.

Observe that Theorems 22 and 7 characterize the candidate pair $(\pi, \eta)$, and allow to find the finite-horizon bounds, but do not address long-run optimality. This stronger property, in fact, holds only under parameter restrictions and is now studied in detail for a single state. In this case, the linear diffusion yields an extension of the models in Kim and Omberg (1996) and Wachter (2002),

$$
\left\{\begin{array}{l}
d R_{t}=\left(\sigma \nu_{0}+b \sigma \nu_{1} Y_{t}\right) d t+\sigma d Z_{t}, \\
d Y_{t}=-b Y_{t} d t+d W_{t}, \\
d\langle R, Y\rangle_{t}=\rho d t \\
r\left(Y_{t}\right)=r_{0} .
\end{array}\right.
$$

The constants are the same as in (54) except that here $\mu_{0}=\sigma \nu_{0}, \mu_{1}=b \sigma \nu_{1}$ where $\nu_{0}, \nu_{1} \in \mathbb{R}^{n}$, for ease of notation. Note that $a=1$ and $r_{1}=0$. The Riccati equation from (56) is

$$
\delta^{-1} v_{1}^{2}+2 b\left(1+q \rho^{\prime} \nu_{1}\right) v_{1}-q b^{2} \nu_{1}^{\prime} \nu_{1}=0
$$

for as in (31). Under Assumption 21, for $p<0$, the solution $v_{1}, v_{0}, \lambda$ from Theorem 22 is

$$
\begin{aligned}
& v_{1}=\delta b\left(\sqrt{\Theta}-\left(1+q \rho^{\prime} \nu_{1}\right)\right), \\
& v_{0}=q \delta \rho^{\prime} \nu_{0}-\frac{1}{\sqrt{\Theta}}\left(q \nu_{1}^{\prime} \nu_{0}+q \delta \rho^{\prime} \nu_{0}\left(1+q \rho^{\prime} \nu_{1}\right)\right), \\
& \lambda=p r_{0}-\frac{1}{2} q \nu_{0}^{\prime} \nu_{0}+\frac{1}{2} \delta^{-1} v_{0}^{2}-q v_{0} \rho^{\prime} \nu_{0}-\frac{1}{2} v_{1},
\end{aligned}
$$

where

$$
\Theta=\left(1+q \rho^{\prime} \nu_{1}\right)^{2}+\delta^{-1} q \nu_{1}^{\prime} \nu_{1} .
$$

The candidate long-run optimal pair $(\pi, \eta)$ is affine in the state variable

$$
\begin{aligned}
& \pi(y)=\frac{1}{1-p} \Sigma^{-1}\left(\mu(y)+v_{0} \sigma \rho-v_{1} y \sigma \rho\right), \\
& \eta(y)=v_{0}-v_{1} y
\end{aligned}
$$

and the dynamics of $(Y, R)$ under the candidate long-run martingale measure are

$$
\left\{\begin{array}{l}
d R_{t}=\sigma d Z_{t}, \\
d Y_{t}=\left(-b Y_{t}-\rho^{\prime} \sigma^{-1} \mu+\left(1-\rho^{\prime} \rho\right)\left(v_{0}-v_{1} Y_{t}\right)\right) d t+d W_{t} .
\end{array}\right.
$$

This pair $(\pi, \eta)$ is indeed long-run optimal but only under a parameter restriction. 
Proposition 23. Let Assumption 21 hold and let $p<0,(\pi, \eta)$ from (63) is long-run optimal if

$$
\left(1-2 q \rho^{\prime} \rho\right) \sqrt{\Theta}+\left(1+q \rho^{\prime} \nu_{1}\right)>0 .
$$

In the case $\nu_{1}=-\kappa \rho$ for $\kappa>0$, which still nests the models of Kim and Omberg (1996) and Wachter (2002), the parameter restriction in (65) simplifies as follows.

Corollary 24. Let Assumption 21 hold. For $p<0$ and $\nu_{1}=-\kappa \rho$ for $\kappa \in \mathbb{R}$. If $0<q \rho^{\prime} \rho \leq 1 / 4$ then long-run optimality holds for all $\kappa$. For $1 / 4<$ $q \rho^{\prime} \rho<1$ long-run optimality holds if

$$
\kappa<\frac{2}{4 q \rho^{\prime} \rho-1} .
$$

Thus long-run optimality requires a joint restriction on preferences $(q)$ and price dynamics $\left(\rho^{\prime} \rho\right.$ and $\left.\kappa\right)$. First, since $q \rho^{\prime} \rho<1$, long-run optimality always holds if $\kappa<\frac{2}{3}$, that is, if risk premia have low covariation with changes in state variables. If this condition is not satisfied, long-run optimality still holds regardless of the level of incompleteness $\left(\rho^{\prime} \rho\right)$ if risk aversion is sufficiently low $\left(q<\frac{1}{4}\right)$. Conversely, if the market is sufficiently incomplete $\left(\rho^{\prime} \rho<\frac{1}{4}\right)$, the restriction holds regardless of preferences. Hence, a violation of long-run optimality requires a high sensitivity of risk premia, high risk aversion and a nearly complete market.

When long-run optimality fails, it does so at different scales, depending on parameters. The next proposition studies this phenomenon in the case $\kappa=1$, which corresponds to a continuous time version of the model of Summers (1986).

Proposition 25. Let Assumption 21 hold. For $p<0$ and $\kappa=1$ from Corollary 24, long-run optimality holds if $q \rho^{\prime} \rho<\frac{3}{4}$. If $q \rho^{\prime} \rho \geq \frac{3}{4}$, long-run optimality fails. In particular:

(i) if $q \rho^{\prime} \rho>\frac{3}{4}$, there exists a finite $T$ such that $\frac{1}{p} E\left[\left(X_{T}^{\pi}\right)^{p}\right]=-\infty$;

(ii) if $q \rho^{\prime} \rho=\frac{3}{4}$ and $\nu_{0}=0$, the certainty equivalent loss is bounded;

(iii) if $q \rho^{\prime} \rho=\frac{3}{4}$ and $\nu_{0} \neq 0$, the certainty equivalent loss diverges to $\infty$.

4.1.1. Calibration. A calibration to real data shows that long-run optimality holds for typical levels of risk aversion, in the model with one asset and one state considered by Barberis (2000) and Wachter (2002). The state variable represents the dividend yield and the asset is an equity index. In the notation of this section, they use the set of parameter values (in monthly units) $\rho=-0.935, r=0.14 \%, \sigma=4.36 \%, \nu_{0}=0.0788, \kappa=0.8944$, 

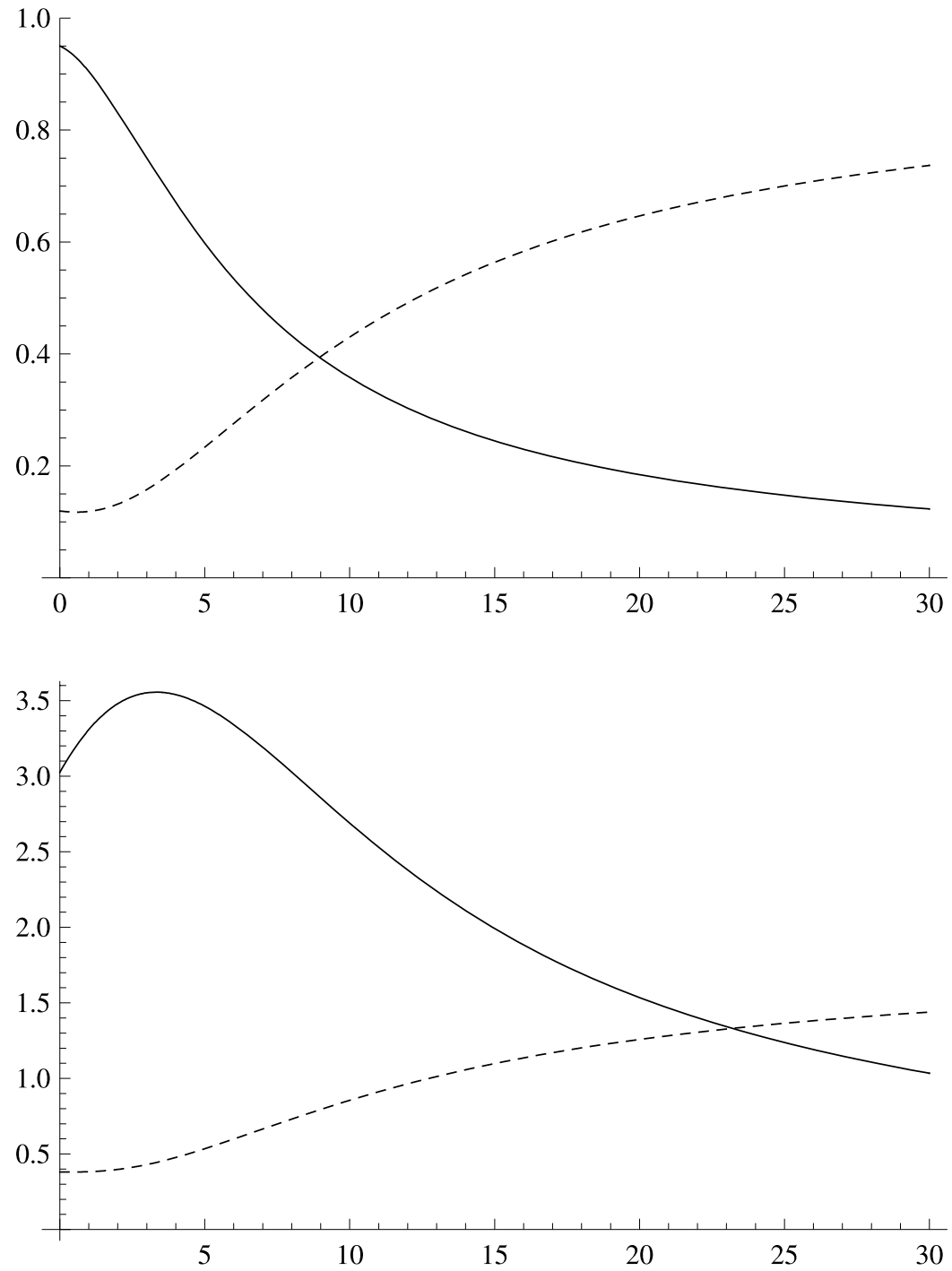

FIG. 1. Annualized certainty equivalent loss bound (in percentage points) as a function of the horizon (in years) of the myopic component (dashed line) and long-run optimal (solid line) portfolios. Both plots are obtained by equation (19) setting $\eta$ equal to the long-run optimal risk premium, and $\pi$ equal to the long-run optimal portfolio (solid line) and to the myopic portfolio $\frac{1}{1-p} \Sigma^{-1} \mu$ (dashed line). Risk aversion is equal to two $(p=-1$, top) and to five $(p=-4$, bottom).

$b=0.0226$. Then, condition (66) is satisfied for $p>-12.4$, that is, for riskaversion less than 13.4 .

Figure 1 compares the finite-horizon performance of the long-run optimal portfolio to the one of its myopic component. The plots show the estimates 
of the correponding upper bounds in (19): the myopic component prevails in the short run but its performance progressively deteriorates as the horizon increases. The break-even horizon significantly increases with risk-aversion, passing from nine years for a risk-aversion of two, to twenty-three years for a risk-aversion of five. Also, the magnitude of the certainty equivalent loss increases with risk aversion: the differences are within one percentage point for a risk-aversion of two but increase to three precentage points for a risk-aversion of five.

4.2. Stochastic drifts, volatilities and interest rates. The next model features a single state variable following the square-root diffusion of Feller (1951), which simultaneously affects the interest rate [Cox, Ingersoll and Ross (1985)], the volatilities of risky assets and their drifts. Note that the model is neither affine nor quadratic (due to the presence of the term with $\nu_{0}$ ) and yet the long-run solution admits a simple expression

$$
\left\{\begin{array}{l}
d R_{t}=\left(\sigma \nu_{0}+\sigma \nu_{1} Y_{t}\right) d t+\sqrt{Y_{t}} \sigma d Z_{t}, \\
d Y_{t}=b\left(\theta-Y_{t}\right) d t+a \sqrt{Y_{t}} d W_{t}, \\
d\langle R, Y\rangle_{t}=\rho d t \\
r\left(Y_{t}\right)=r_{0}+r_{1} Y_{t}
\end{array}\right.
$$

where $\sigma \in \mathbb{R}^{n \times n} ; \nu_{0}, \nu_{1} \in \mathbb{R}^{n} ; b, \theta, a \in \mathbb{R}, \rho \in \mathbb{R}^{n}$ and $r_{0}, r_{1} \in \mathbb{R}$. The parameters satisfy the following.

Assumption 26. $b, \theta, a, r_{1} \geq 0$ and $b \theta>\frac{1}{2} a^{2}$.

Assumption 26 ensures that the state variable $Y$ remains strictly positive, thereby satisfying Assumption 2 with $E=(0, \infty)$. Guessing a form of the solution $v(y)=v_{0} \log y+v_{1} y$, the main $\mathrm{ODE}(22)$ becomes

$$
\begin{aligned}
p r_{0}+ & p r_{1} y-\frac{q}{2}\left(\nu_{0}^{\prime}+y \nu_{1}^{\prime}\right) \frac{1}{y}\left(\nu_{0}+\nu_{1} y\right)+\frac{1}{2}\left(\frac{v_{0}}{y}+v_{1}\right) a^{2} \delta^{-1} y\left(\frac{v_{0}}{y}+v_{1}\right) \\
& +\left(\frac{v_{0}}{y}+v_{1}\right)\left(b \theta-q a \rho^{\prime} \nu_{0}-\left(b+q a \rho^{\prime} \nu_{1}\right) y\right)-\frac{1}{2} a^{2} \frac{v_{0}}{y}=\lambda .
\end{aligned}
$$

Multiplying the above equation by $y$ and setting the constant, linear and quadratic terms to zero leads to four candidate solutions, corresponding to any combination of signs in the terms $\pm \sqrt{\Theta}$ and $\pm \sqrt{\Lambda}$ below:

$$
\begin{aligned}
v_{1} & =\frac{\delta}{a^{2}}\left(b+q a \rho^{\prime} \nu_{1} \pm \sqrt{\Theta}\right) \\
v_{0} & =\frac{\delta}{a^{2}}\left(-\left(c-q a \rho^{\prime} \nu_{0}\right) \pm \sqrt{\Lambda}\right) \\
\lambda & =p r_{0}-q \nu_{0}^{\prime} \nu_{1}+\frac{a^{2}}{\delta} v_{0} v_{1}-v_{0}\left(b+q a \rho^{\prime} \nu_{1}\right)+v_{1}\left(b \theta-q a \rho^{\prime} \nu_{0}\right)
\end{aligned}
$$


with

$$
\begin{aligned}
& c=b \theta-\frac{1}{2} a^{2}, \\
& \Theta=\left(b+q a \rho^{\prime} \nu_{1}\right)^{2}+\frac{a^{2}}{\delta}\left(q \nu_{1}^{\prime} \nu_{1}-2 p r_{1}\right), \\
& \Lambda=\left(c-q a \rho^{\prime} \nu_{0}\right)^{2}+\frac{a^{2}}{\delta} q \nu_{0}^{\prime} \nu_{0} .
\end{aligned}
$$

When $p<0, r_{1}>0$,

$$
\begin{aligned}
& \Theta>\left(b+q a \rho^{\prime} \nu_{1}\right)^{2}>0, \\
& \Lambda>\left(c-q a \rho^{\prime} \nu_{0}\right)^{2}>0 .
\end{aligned}
$$

Under $\hat{P}, Y$ has again four possible dynamics:

$$
d Y_{t}=\left(\frac{1}{2} a^{2} \pm \sqrt{\Lambda} \pm \sqrt{\Theta} Y_{t}\right) d t+a \sqrt{Y_{t}} d W_{t} .
$$

The choice of $-\sqrt{\Theta}$ and $\sqrt{\Lambda}$ ensures that $Y$ satisfies Assumption 2 under $\hat{P}^{y}$ and is $\hat{P}^{y}$-tight in $(0, \infty)$ for each $y \in(0, \infty)$. The latter statement follows by the positivity of $\Theta, \Lambda$ [see Pinsky (1995), Corollary 5.1.11, and the discussion immediately after Assumption 12]. Thus the candidate optimizer is

$$
\begin{aligned}
v_{1} & =\frac{\delta}{a^{2}}\left(b+q a \rho^{\prime} \nu_{1}-\sqrt{\Theta}\right), \\
v_{0} & =\frac{\delta}{a^{2}}\left(-\left(c-q a \rho^{\prime} \nu_{0}\right)+\sqrt{\Lambda}\right), \\
\lambda & =p r_{0}-q \nu_{0}^{\prime} \nu_{1}+\frac{a^{2}}{\delta} v_{0} v_{1}-v_{0}\left(b+q a \rho^{\prime} \nu_{1}\right)+v_{1}\left(b \theta-q a \rho^{\prime} \nu_{0}\right) .
\end{aligned}
$$

The candidate long-run optimal policies $(\pi, \eta)$ are

$$
\pi(y)=\frac{1}{1-p} \Sigma^{-1}\left(\mu(y)+\sigma \rho a\left(v_{0}+v_{1} y\right)\right), \quad \eta(y)=\frac{v_{0}}{y}+v_{1}
$$

and the candidate long-run martingale measure is

$$
\left\{\begin{aligned}
d R_{t}= & \sqrt{Y_{t}} \sigma d Z_{t} \\
d Y_{t}= & \left(b\left(\theta-Y_{t}\right)-q a\left(\rho^{\prime} \nu_{0}+\rho^{\prime} \nu_{1} Y_{t}\right)+a^{2}\left(1-\rho^{\prime} \rho\right)\left(v_{0}+v_{1} Y_{t}\right)\right) d t \\
& +a \sqrt{Y_{t}} d W_{t} .
\end{aligned}\right.
$$

Long-run optimality obtains under the following conditions.

Proposition 27. Let Assumption 26 hold. For $p<0$, long-run optimality holds if

$$
\begin{aligned}
& \left(1-2 q \rho^{\prime} \rho\right) \sqrt{\Lambda}+\left(c-q a \rho^{\prime} \nu_{0}\right)>0 \\
& \left(1-2 q \rho^{\prime} \rho\right) \sqrt{\Theta}+\left(b+q a \rho^{\prime} \nu_{1}\right)>0 .
\end{aligned}
$$


The main economic message of this parametric restriction is the same as in the previous example. Long-run optimality holds if either one of the following conditions is satisfied: the covariation of risk premia with state shocks is small $\left(a \rho^{\prime} \nu_{0}, a \rho^{\prime} \nu_{1} \approx 0\right)$, the market is sufficiently incomplete $\left(\rho^{\prime} \rho \ll 1\right)$ or risk aversion is low $(1-p \ll \infty)$.

5. Conclusion. Long-run analysis is a tractable and yet nontrivial framework for dynamic portfolio choice and derivatives pricing in incomplete markets, and yields simple expressions for portfolios and risk premia. Long-run policies admit closed-form solutions even in cases in which finite-horizon policies do not, and the finite-horizon performance of long-run policies has a simple expression.

Long-run optimality entails that the certainty equivalent loss vanishes for long horizons and requires some joint restrictions on preferences and asset dynamics. It does not hold at the intersection of three extreme cases: risk premia highly co-varying with state shocks, a nearly complete market and high risk aversion. Otherwise, long-run optimality holds and time-homogeneous portfolios are approximately optimal for long horizons.

\section{APPENDIX A: PROOF OF SECTION 1}

Proof of Lemma 5. Denote by $q=\frac{p}{p-1}$. In the case $0<p<1$, Hölder's inequality with $\tilde{p}=\frac{1}{p}$ and $\tilde{q}=\frac{\tilde{p}}{\tilde{p}-1}=\frac{1}{1-p}$ yields

$$
\begin{aligned}
E_{P}\left[X^{p}\right] & =E_{P}\left[(X M)^{p} M^{-p}\right] \leq E_{P}\left[(X M)^{p \tilde{p}}\right]^{1 / \tilde{p}} E_{P}\left[M^{-p \tilde{q}}\right]^{1 / \tilde{q}} \\
& =E_{P}[X M]^{1 / \tilde{p}} E_{P}\left[M^{q}\right]^{1-p} \leq E_{P}\left[M^{q}\right]^{1-p},
\end{aligned}
$$

because $E_{P}[X M] \leq 1$, and the claim follows dividing by $p>0$. If $p<0$ $(0<q<1)$ Hölder's inequality with $\tilde{p}=\frac{1}{1-q}, \tilde{q}=\frac{1}{q}$ yields

$$
\begin{aligned}
E_{P}\left[M^{q}\right]^{1-p} & =E_{P}\left[(X M)^{q} X^{-q}\right]^{1-p} \leq E_{P}\left[(X M)^{q \tilde{q}}\right]^{(1-p) / \tilde{q}} E_{P}\left[X^{-q \tilde{p}}\right]^{(1-p) / \tilde{p}} \\
& =E_{P}[X M]^{-p} E_{P}\left[X^{p}\right] \leq E_{P}\left[X^{p}\right]
\end{aligned}
$$

and the claim now follows dividing by $p<0$. In both cases, the inequality becomes an equality when $E_{P}[X M]=1$ and $X^{p-1}$ is proportional to $M$.

\section{APPENDIX B: PROOFS OF SECTION 2}

Proof of Theorem 7. Since the Brownian motions $Z$ and $W$ are partially correlated, the following orthogonal decomposition holds (see the discussion following Assumption 2):

$$
d Z_{t}=\rho\left(Y_{t}\right) d W_{t}+\bar{\rho}\left(Y_{t}\right) d B_{t},
$$


where $B=\left(B^{1}, \ldots, B^{n}\right)$ is a $n$-dimensional Brownian motion independent of $W$ and the matrix $\bar{\rho}(y)$ is defined by the identity $\left(\rho \rho^{\prime}\right)(y)+\left(\bar{\rho} \bar{\rho}^{\prime}\right)(y)=I_{n}$. For $t \geq 0$, define the process $D$ by

$$
\begin{aligned}
D_{t}=\mathcal{E}\left(\int _ { 0 } \left(-q \Upsilon^{\prime} \Sigma^{-1} \mu\right.\right. & \left.+\left(A-q \Upsilon^{\prime} \Sigma^{-1} \Upsilon\right) \nabla v\right)^{\prime}\left(a^{\prime}\right)^{-1} d W_{t} \\
& \left.-\int_{0}^{\cdot} q\left(\Sigma^{-1} \mu+\Sigma^{-1} \Upsilon \nabla v\right)^{\prime} \sigma \bar{\rho} d B_{t}\right)_{t}
\end{aligned}
$$

By assumption, the operators associated to the models $P$ and $\hat{P}$ satisfy Assumption 2. Since Assumption 1 also holds, Cheridito, Filipović and Yor [(2005), Theorem 2.4, Remark 2.4.2] implies that for each $y \in E$ and $t \geq 0, P^{y}$ and $\hat{P}^{y}$ are equivalent on $\mathcal{F}_{t}$ with

$$
\left.\frac{d \hat{P}^{y}}{d P^{y}}\right|_{\mathcal{F}_{t}}=D_{t} .
$$

Thus, $\left(D_{t}, \mathcal{F}_{t}\right)_{t \geq 0}$ is a $P^{y}$-martingale. With this notation, it suffices to prove that given the solution pair $(v, \lambda)$ to $(22)$, with $\pi, \eta$ as in $(24)$, the following $P^{y}$ almost sure identities hold:

$$
\begin{aligned}
\left(X_{T}^{\pi}\right)^{p} & =e^{\lambda T+v(y)-v\left(Y_{T}\right)} D_{T}, \\
\left(M_{T}^{\eta}\right)^{q} & =e^{(1 /(1-p))\left(\lambda T+v(y)-v\left(Y_{T}\right)\right)} D_{T} .
\end{aligned}
$$

Indeed, if (75) and (76) hold, then (25) and (26) follow by taking expectations with respect to $P^{y}$. Consider first (75). Passing to logarithms, it suffices to prove that

$$
p \log X_{T}^{\pi}-\log D_{T}=\lambda T+v(y)-v\left(Y_{T}\right) .
$$

The first term on the left-hand side of (77) is

$$
p \log X_{T}^{\pi}=\int_{0}^{T}\left(p r+p \pi^{\prime} \mu-\frac{1}{2} p \pi^{\prime} \Sigma \pi\right) d t+\int_{0}^{T} p \pi^{\prime} \sigma d Z_{t} .
$$

Substituting $\pi=\frac{1}{1-p} \Sigma^{-1}(\mu+\Upsilon \nabla v)$, the decomposition $Z=\rho W+\bar{\rho} B$ and collecting terms

$$
\begin{aligned}
p \log X_{T}^{\pi}= & \int_{0}^{T}\left(p r-\frac{1}{2} q(1+q) \mu^{\prime} \Sigma^{-1} \mu-q^{2} \mu^{\prime} \Sigma^{-1} \Upsilon \nabla v\right. \\
& \left.+\frac{1}{2} q(1-q) \nabla v^{\prime} \Upsilon^{\prime} \Sigma^{-1} \Upsilon \nabla v\right) d t \\
& -q \int_{0}^{T}(\mu+\Upsilon \nabla v)^{\prime} \Sigma^{-1} \sigma \rho d W_{t}-q \int_{0}^{T}(\mu+\Upsilon \nabla v)^{\prime} \Sigma^{-1} \sigma \bar{\rho} d B_{t} .
\end{aligned}
$$


The second term in the left-hand side of (77) follows from (73),

$$
\begin{aligned}
\log D_{T}= & \int_{0}^{T}\left(-\frac{1}{2} q^{2} \mu^{\prime} \Sigma^{-1} \mu+q(1-q) \mu^{\prime} \Sigma^{-1} \Upsilon \nabla v\right. \\
& \left.\quad-\frac{1}{2} \nabla v^{\prime}\left(A+\left(q^{2}-2 q\right) \Upsilon^{\prime} \Sigma^{-1} \Upsilon\right) \nabla v\right) d t \\
& +\int_{0}^{T}\left(\nabla v^{\prime} a-q(\mu+\Upsilon \nabla v)^{\prime} \Sigma^{-1} \sigma \rho\right) d W_{t} \\
& -q \int_{0}^{T}(\mu+\Upsilon \nabla v) \Sigma^{-1} \sigma \bar{\rho} d B_{t} .
\end{aligned}
$$

Subtracting (79) from (78) yields

$$
\begin{aligned}
p \log X_{T}^{\pi}-\log D_{T} & \\
= & \int_{0}^{T}\left(p r-\frac{1}{2} q \mu^{\prime} \Sigma^{-1} \mu-q \mu^{\prime} \Sigma^{-1} \Upsilon \nabla v+\frac{1}{2} \nabla v^{\prime}\left(A-q \Upsilon^{\prime} \Sigma^{-1} \Upsilon\right) \nabla v\right) d t \\
& -\int_{0}^{T} \nabla v^{\prime} a d W_{t} .
\end{aligned}
$$

Now, Itô's formula allows us to substitute

$$
-\int_{0}^{T} \nabla v^{\prime} a d W_{t}=v(y)-v\left(Y_{T}\right)+\int_{0}^{T} \nabla v^{\prime} b d t+\frac{1}{2} \int_{0}^{T} \operatorname{tr}\left(A D^{2} v\right) d t
$$

and the claim (77) follows by recalling that $(v, \lambda)$ solves $(22)$.

Consider now the equality (76). Again, by taking logarithms it suffices to show that

$$
q \log M_{T}^{\eta}-\log D_{T}=\frac{1}{1-p}\left(\lambda T+v(y)-v\left(Y_{T}\right)\right) .
$$

The first term in the left-hand side is equal to (plugging $\eta=\nabla v$ and $Z=$ $\rho W+\bar{\rho} B)$

$$
\begin{aligned}
q \log M_{T}^{\eta}= & -q \int_{0}^{T} r d t+q \log \mathcal{E}\left(-\int_{0}\left(\Sigma^{-1} \mu+\Sigma^{-1} \Upsilon \eta\right)^{\prime} \sigma d Z_{t}\right. \\
& \left.+\int_{0} \eta^{\prime} a d W_{t}\right)_{T} \\
= & -q \int_{0}^{T}\left(r+\frac{1}{2} \mu^{\prime} \Sigma^{-1} \mu+\frac{1}{2} \nabla v^{\prime}\left(A-\Upsilon^{\prime} \Sigma^{-1} \Upsilon\right) \nabla v\right) d t \\
& +q \int_{0}^{T}\left(\nabla v^{\prime} a-(\mu+\Upsilon \nabla v)^{\prime} \Sigma^{-1} \sigma \rho\right) d W_{t} \\
& -q \int_{0}^{T}(\mu+\Upsilon \nabla v) \Sigma^{-1} \sigma \bar{\rho} d B_{t}
\end{aligned}
$$


and subtracting (79) yields

$$
\begin{aligned}
q \log M_{T}^{\eta}-\log D_{T}= & \frac{1}{1-p} \int_{0}^{T}\left(p r-\frac{1}{2} q \mu^{\prime} \Sigma^{-1} \mu-q \mu^{\prime} \Sigma^{-1} \Upsilon \nabla v\right. \\
& \left.+\frac{1}{2} \nabla v^{\prime}\left(A-q \Upsilon^{\prime} \Sigma^{-1} \Upsilon\right) \nabla v\right) d t \\
& -\frac{1}{1-p} \int_{0}^{T} \nabla v^{\prime} a d W_{t} .
\end{aligned}
$$

As in the previous case, (81) now follows by substituting (80) and recalling that $(v, \lambda)$ solves $(22)$.

The proof of Theorem 9 requires two lemmas.

LEMmA 28. Let $\phi \in C(E ; \mathbb{R}), \phi>0$ and let $\left(\mu_{T}\right)_{T \geq 0}$ be a tight family of probability measures on $(E, \mathcal{B}(E))$. Then

$$
\liminf _{T \uparrow \infty} \frac{1}{T} \log \int \phi d \mu_{T} \geq 0
$$

Proof. By monotonicity, it suffices to prove the result for $\phi$ bounded. Let $\left(t_{n}\right)_{n \geq 1}$ be an increasing sequence satisfying $t_{n} \uparrow \infty$ and

$$
\liminf _{T \uparrow \infty} \frac{1}{T} \log \int_{E} \phi d \mu_{T}=\lim _{n \rightarrow \infty} \frac{1}{t_{n}} \log \int_{E} \phi d \mu_{t_{n}} .
$$

Since $\phi$ is bounded above, the limit in (83) is nonpositive. Since the measures $\left(\mu_{T}\right)_{T \geq 0}$ are tight, they are relatively compact with respect to the topology of weak convergence. Thus, up to a subsequence, there exists a probability measure $\mu$ on $E$ such that

$$
\lim _{n \rightarrow \infty} \int_{E} \phi d \mu_{t_{n}}=\int_{E} \phi d \mu \in(0, \infty)
$$

because $\phi$ is continuous, bounded and positive. Thus, for any $0<\varepsilon<\int_{E} \phi d \mu$, there is a $n_{\varepsilon}$ such that $n \geq n_{\varepsilon}$ implies

$$
\frac{1}{t_{n}} \log \int_{E} \phi d \mu_{t_{n}} \geq \frac{1}{t_{n}} \log \left(\int_{E} \phi d \mu-\varepsilon / 2\right) \text {. }
$$

Hence, taking $n \uparrow \infty$ it follows that the limit in (83) is indeed 0 .

Lemma 29. Let $\left(Q^{y}\right)_{y \in E}$ be a solution to the martingale problem for the operator $L$ on $E$, where

$$
L=\frac{1}{2} \sum_{i, j=1}^{k} \Sigma(y)^{i j} \frac{\partial^{2}}{\partial y_{i} \partial y_{j}}+\sum_{i=1}^{k} \mu(y)^{i} \frac{\partial}{\partial y_{i}} .
$$

If $f \in C^{2}(E, \mathbb{R}), f>0$, then $E_{Q}^{y}\left[f\left(Y_{T}\right)\right] \leq f(y)+\left(0 \vee \sup _{E} L f\right) T$. 
Proof. Let $f \in C^{2}(E, \mathbb{R}), f>0$. Since $\left(Q^{y}\right)_{y \in E}$ solves the martingale problem for $L$ on $E$, the process

$$
f\left(Y_{T}\right)-\int_{0}^{T}(L f)\left(Y_{t}\right) d t
$$

is a local martingale under $Q^{y}$. Let $\left(\tau_{n}\right)_{n \geq 1}$ be a reducing sequence of stopping times for this local martingale. Then

$$
E_{Q}^{y}\left[f\left(Y_{T \wedge \tau_{n}}\right)\right]=f(y)+E_{Q}^{y}\left[\int_{0}^{T \wedge \tau_{n}}(L f)\left(Y_{t}\right) d t\right] \leq f(y)+\left(0 \vee \sup _{E}(L f)\right) T
$$

and the thesis follows by Fatou's lemma, taking $n \uparrow \infty$.

Proof of Theorem 9. Applying the inequality in (17) to equations (25) and (26) from Theorem 7 gives

$$
\begin{aligned}
0 & \leq \liminf _{T \rightarrow \infty} \frac{1}{p}\left(\frac{1}{T} \log E_{P}^{y}\left[\left(M_{T}^{\eta}\right)^{q}\right]^{1-p}-\frac{1}{T} \log E_{P}^{y}\left[\left(X_{T}^{\pi}\right)^{p}\right]\right) \\
& \leq \limsup _{T \rightarrow \infty} \frac{1}{p T} \log E_{P}^{y}\left[\left(M_{T}^{\eta}\right)^{q}\right]^{1-p}-\liminf _{T \rightarrow \infty} \frac{1}{p T} \log E_{P}^{y}\left[\left(X_{T}^{\pi}\right)^{p}\right] \\
& =\limsup _{T \rightarrow \infty} \frac{1-p}{p T} \log E_{\hat{P}}^{y}\left[e^{-(1 /(1-p)) v\left(Y_{T}\right)}\right]-\liminf _{T \rightarrow \infty} \frac{1}{p T} \log E_{\hat{P}}^{y}\left[e^{-v\left(Y_{T}\right)}\right] .
\end{aligned}
$$

Thus it is sufficient to prove for $0<p<1$ that

$$
\begin{aligned}
& \limsup _{T \rightarrow \infty} \frac{1}{T} \log E_{\hat{P}}^{y}\left[\exp \left(-\frac{1}{1-p} v\left(Y_{T}\right)\right)\right] \leq 0, \\
& \liminf _{T \rightarrow \infty} \frac{1}{T} \log E_{\hat{P}}^{y}\left[\exp \left(-v\left(Y_{T}\right)\right)\right] \geq 0
\end{aligned}
$$

and for $p<0$ that

$$
\begin{aligned}
& \liminf _{T \rightarrow \infty} \frac{1}{T} \log E_{\hat{P}}^{y}\left[\exp \left(-\frac{1}{1-p} v\left(Y_{T}\right)\right)\right] \geq 0, \\
& \limsup _{T \rightarrow \infty} \frac{1}{T} \log E_{\hat{P}}^{y}\left[\exp \left(-v\left(Y_{T}\right)\right)\right] \leq 0
\end{aligned}
$$

for $p<0$. The lower bounds (87) and (86) follow from the application of Lemma 28 to the functions $\phi=\exp \left(-\frac{1}{1-p} v\right)$ and $\phi=\exp (-v)$, respectively. For the upper bounds, first denote by

$$
L f=\nabla f^{\prime}\left(b-q \Upsilon^{\prime} \Sigma^{-1} \mu+\left(A-q \Upsilon^{\prime} \Sigma^{-1} \Upsilon\right) \nabla v\right)+\frac{1}{2} \operatorname{tr}\left(A D^{2} f\right)
$$

and observe that, for any $\alpha \in \mathbb{R}$,

$$
L\left(e^{\alpha v}\right)=\alpha e^{\alpha v}\left(\nabla v^{\prime}\left(b-q \Upsilon^{\prime} \Sigma^{-1} \mu+\left(A-q \Upsilon^{\prime} \Sigma^{-1} \Upsilon\right) \nabla v\right)\right.
$$




$$
\begin{gathered}
\left.+\frac{1}{2} \operatorname{tr}\left(A D^{2} v\right)+\frac{1}{2} \alpha \nabla v^{\prime} A \nabla v\right) \\
=\alpha e^{\alpha v}\left(\frac{1}{2} \nabla v^{\prime}\left((1+\alpha) A-q \Upsilon^{\prime} \Sigma^{-1} \Upsilon\right) \nabla v+\lambda-p r+\frac{q}{2} \mu^{\prime} \Sigma^{-1} \mu\right),
\end{gathered}
$$

where the second equality follows from (22). For $p<0$, consider $\alpha=-1$,

$$
L\left(e^{-v}\right)=e^{-v}\left(\frac{q}{2} \nabla v^{\prime} \Upsilon^{\prime} \Sigma^{-1} \Upsilon \nabla v-\lambda+p r-\frac{q}{2} \mu^{\prime} \Sigma^{-1} \mu\right) .
$$

Assumption (ii) implies that the right-hand side is bounded by some constant $K$ and Lemma 29 yields

$$
E_{\hat{P}}^{y}\left[e^{-v\left(Y_{T}\right)}\right] \leq e^{-v(y)}+(K \vee 0) T
$$

and hence,

$$
\limsup _{T \rightarrow \infty} \frac{1}{T} \log E_{\hat{P}}^{y}\left[e^{-v\left(Y_{T}\right)}\right] \leq 0 .
$$

Similarly, for $0<p<1$ consider $\alpha=-\frac{1}{1-p}$,

$$
\begin{aligned}
L\left(e^{-(1 /(1-p)) v}\right)= & \frac{1}{1-p} e^{-(1 /(1-p)) v} \\
& \times\left(-\frac{q}{2} \nabla v^{\prime}\left(A-\Upsilon^{\prime} \Sigma^{-1} \Upsilon\right) \nabla v-\lambda+p r-\frac{q}{2} \mu^{\prime} \Sigma^{-1} \mu\right) .
\end{aligned}
$$

Again, the right-hand side is bounded by $K$ and Lemma 29 yields

$$
E_{\hat{P}}^{y}\left[e^{-(1 /(1-p)) v\left(Y_{T}\right)}\right] \leq e^{-(1 /(1-p)) v(y)}+(K \vee 0) T
$$

and the claim follows as in the previous case.

The proofs of Theorems 13 and 18 are obtained by adapting the arguments in Kaise and Sheu (2006) to the present setting. Because the structure of these proofs remains the same, the arguments are not repeated in detail, focusing instead on the necessary modifications.

Henceforth, all references in italics point to Kaise and Sheu (2006). For ease of notation, set

$$
\hat{A}=A-q \Upsilon^{\prime} \Sigma^{-1} \Upsilon, \quad \tilde{b}=b-q \Upsilon^{\prime} \Sigma^{-1} \mu, \quad V=p r-\frac{q}{2} \mu^{\prime} \Sigma^{-1} \mu
$$

and define the quasilinear operator $M$ acting on $f \in C^{2}(E)$ by

$$
M f=\frac{1}{2} \operatorname{tr}\left(A D^{2} f\right)+\frac{1}{2} \nabla f^{\prime} \hat{A} \nabla f+\tilde{b}^{\prime} \nabla f+V
$$

so that (22) becomes $M v=\lambda$. The following results carry over immediately from Kaise and Sheu (2006) and Ladyzhenskaya and Ural'tseva (1968) with only the obvious notational changes. 
LEMma 30 (Lemma 2.4 and the discussion following). Let Assumption 1 hold. Let $\lambda \in \mathbb{R}$. Then:

(i) If, for each $N$ there exists a $v_{N} \in C^{2, \gamma}\left(E_{N}, \mathbb{R}\right)$ satisfying $M v_{N}=\lambda$ in $E_{N}$, then there exists a $v \in C^{2, \gamma}(E, \mathbb{R})$ satisfying $M v=\lambda$.

(ii) If $\lambda_{m} \rightarrow \lambda$ and for each $m$ there is a $v_{m} \in C^{2, \gamma}(E, \mathbb{R})$ solving $M v_{m}=\lambda_{m}$, then there is a $v \in C^{2, \gamma}(E, \mathbb{R})$ solving $M v=\lambda$.

REMARK 31. Note that Lemma 2.4 requires the uniform ellipticity of diffusion matrices in the form of conditions (A1), (A2). Under Assumption 1, these conditions are satisfied on each $E_{N}$ by continuity and positivity. In the present setting, the conclusion of Lemma 2.4 is that, if $v_{N} \in C^{2, \gamma}\left(E_{N}, \mathbb{R}\right)$ solves $M v_{N}=\lambda$ in $E_{N}$, then for each $n$ there exist constants $A_{n}, B_{n}, C_{n}>0$ such that for $N>2 n$

$$
\sup _{E_{n}}\left|\nabla v_{N}\right| \leq \max \left\{A_{n}+B_{n} \lambda, C_{n}\right\} .
$$

Lemma 32 [Theorem 8.4 in Ladyzhenskaya and Ural'tseva (1968)]. Let Assumptions 1 and 11 hold. For any $N$, if there exist functions $f_{1}, f_{2} \in$ $C^{2, \gamma}\left(E_{N+1}, \mathbb{R}\right) \cap C\left(\bar{E}_{N+1}, \mathbb{R}\right)$ satisfying $M f_{1}>\lambda$ and $M f_{2}<\lambda$ in $E_{N}$, then there exists a function $v_{N} \in C^{2, \gamma}\left(E_{N}, \mathbb{R}\right)$ satisfying $M v_{N}=\lambda$.

Proof of Theorem 13. In Kaise and Sheu (2006) Theorem 13 is split into two theorems: Theorem 2.6 shows that $\Lambda=\left[\lambda_{c}, \infty\right)$, while Theorem 3.2

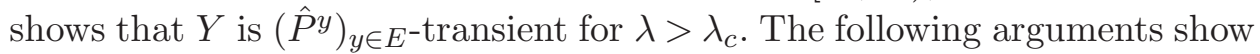
that the conclusions of both Theorem 2.6 and Theorem 3.2 remain valid.

Regarding Theorem 2.6, given Lemmas 30 and 32, it suffices to prove that:

(A) There exists a $\lambda_{0} \in \mathbb{R}$ such that for each $N$ there is a $v_{N} \in C^{2, \gamma}\left(E_{N}, \mathbb{R}\right)$ satisfying $M v_{N}=\lambda_{0}$ in $E_{N}$.

(B) The set $\left\{\lambda \in \mathbb{R}: \exists v \in C^{2, \gamma}(E, \mathbb{R})\right.$ satisfying $\left.M v \geq \lambda\right\}$ is unbounded from above.

(C) $\lambda_{c} \equiv \inf \Lambda>-\infty$.

Indeed, if (A) holds true then Lemma 30 yields a solution $v \in C^{2, \gamma}(E, \mathbb{R})$ to $M v=\lambda_{0}$ proving $\Lambda \neq \varnothing$. Now, let $\lambda \in \Lambda$, let $v \in C^{2, \gamma}(E, \mathbb{R})$ satisfy $M v=\lambda$ and let $\tilde{\lambda}>\lambda$. If $(\mathrm{B})$ holds, then there is a $f_{1} \in C^{2, \gamma}(E, \mathbb{R})$ satisfying $M f_{1}>\tilde{\lambda}$. Thus, with $f_{2}=v$, Lemma 32 applies for any $N$ and hence, by Lemma 30, it follows that $\tilde{\lambda} \in \Lambda$. Thus, $\lambda \in \Lambda \Rightarrow[\lambda, \infty) \subseteq \Lambda$. By (C) it follows that $\Lambda$ is bounded from below and by Lemma 30 it follows that $\Lambda$ is closed. Thus, $\Lambda=\left[\lambda_{c}, \infty\right)$ is the desired result.

Therefore, it remains to prove (A), (B) and (C). First, note that with $a, \sigma$ the unique symmetric, positive definite square roots of $A$ and $\Sigma$, respectively, it follows that

$$
\hat{A}=a\left(1_{k}-q \rho^{\prime} \rho\right) a=(1-q) a a+q a\left(1_{k}-\rho^{\prime} \rho\right) a .
$$


By construction $1_{n}-\rho \rho^{\prime} \geq 0$ and hence, $1_{k}-\rho^{\prime} \rho \geq 0$. Thus, setting

$$
c=\left\{\begin{array}{ll}
1-q, & p<0, \\
1, & 0<p<10,
\end{array} \quad \bar{c}= \begin{cases}1, & p<0, \\
1-q, & 0<p<1,\end{cases}\right.
$$

it follows that $c, \bar{c}>0$ are such that on $E$

$$
c A \leq \hat{A} \leq \bar{c} A .
$$

Define the linear operators $L^{c}$ and $L^{\bar{c}}$ acting on $f \in C^{2}(E)$ by

$$
L^{c} f=\frac{1}{2} \operatorname{tr}\left(A D^{2} f\right)+\tilde{b}^{\prime} \nabla f+c V f, \quad L^{\bar{c}} f=\frac{1}{2} \operatorname{tr}\left(A D^{2} f\right)+\tilde{b}^{\prime} \nabla f+\bar{c} V f .
$$

Let $\lambda^{*}$ and $\bar{\lambda}^{*}$ denote the generalized principal eigenvalue for $L^{c}$ and $L^{\bar{c}}$ on $E$ [Pinsky (1995), Chapter 4.3]. Assumptions 1, 11 and 12 imply that $\lambda^{*}$, $\bar{\lambda}^{*} \in \mathbb{R}$. By (91), it follows that

$$
\frac{1}{c g} L^{c} g \leq M f \leq \frac{1}{\bar{c} \bar{g}} L^{\bar{c}} \bar{g},
$$

where $g=e^{c f}$ and $\bar{g}=e^{\bar{c} f}$. For any $\beta>\max \left[\lambda^{*}, \bar{\lambda}^{*}, 1\right]$, let $\lambda=\frac{\beta}{2}\left(\frac{1}{c}+\frac{1}{\bar{c}}\right)$. By construction there exist $g, \bar{g} \in C^{2, \gamma}(E), g, \bar{g}>0$ satisfying $L^{c} g=\beta g$ and $L^{\bar{c}} \bar{g}=\beta \bar{g}$ in $E$. Set $f_{1}=\frac{1}{c} \log g$. By (92), it follows that on $E$

$$
M f_{1}-\lambda \geq \frac{1}{c g} L^{c} g-\lambda=\frac{\beta}{2}\left(\frac{1}{c}-\frac{1}{\bar{c}}\right)>0 .
$$

Similarly, setting $f_{2}=\frac{1}{\bar{c}} \log \bar{g}$ and using (92) it follows that on $E$

$$
M f_{2}-\lambda \leq \frac{1}{\bar{c} \bar{g}} L^{\bar{c}} \bar{g}-\lambda=\frac{\beta}{2}\left(\frac{1}{\bar{c}}-\frac{1}{c}\right)<0 .
$$

Therefore, (A) holds by first applying Lemma 32 and then Lemma 30(i) for $\lambda_{0}=\lambda$. Furthermore, since $\beta$ can be taken arbitrarily large, (B) holds as well. Regarding (C), let $\lambda \in \Lambda$ and let $v$ be the associated function solving $M v=\lambda$ and let $g=e^{c v}$. By $(92)$

$$
\lambda=\sup _{E}(M v) \geq \frac{1}{c} \sup _{E}\left(\frac{L^{c} g}{g}\right) \geq \frac{1}{c} \inf _{\substack{g \in C^{2}(E, \mathbb{R}) \\ g>0}} \sup _{E}\left(\frac{L^{c} g}{g}\right)=\frac{\lambda^{*}}{c},
$$

where the last equality follows by Pinsky (1995), Theorem 4.4.5. Thus $\lambda_{c}>$ $-\infty$.

Regarding Theorem 3.2, note that by (91), Remark 2.1 holds for $c, \bar{c}$ and the argument in Lemma 3.1 carries over exactly, up to obvious changes in notation.

Before proving Theorem 18, the following definitions and results are needed from the theory of large deviations for occupancy times of diffusions. Let 
Assumptions 1, 11 and 12 hold. To make the dependence upon $\lambda$ specific, for $\lambda \in \Lambda$ let $\left(\hat{P}^{\lambda, y}\right)_{y \in E}$ be the measure in Theorem 7 and let $\hat{L}^{\lambda}$ be the operator associated to $\left(\hat{P}^{\lambda, y}\right)_{y \in E}$. Let $M_{1}(E)$ denote the space of Borel probability measures on $E$. Define the function $I^{\lambda}: M_{1}(E) \mapsto \mathbb{R}$ by

$$
I^{\lambda}(\mu)=-\inf _{u \in \mathcal{U}} \int_{E} \frac{\hat{L}^{\lambda} u}{u} d \mu
$$

where

$$
\mathcal{U}=\left\{u \in C^{2}(E, \mathbb{R}) \mid u(x) \geq \varepsilon_{u}>0, \frac{\hat{L}^{\lambda} u}{u} \text { bounded }\right\} .
$$

It is clear that $I^{\lambda}$ is nonnegative $(u=1)$ and lower semi-continuous with respect to the weak topology on $M_{1}(E)\left(\hat{L}^{\lambda} u / u\right.$ is bounded $)$. Set $\tau_{n}=\inf \{t \geq$ $\left.0: Y_{t} \in E_{n}^{c}\right\}$ and $\tau=\lim _{n \uparrow \infty} \tau_{n}$. Denote by $\mu_{T}$ the occupation measure for $Y$ on $\{T<\tau\}$, which satisfies

$$
\mu_{T}(B)=\frac{1}{T} \int_{0}^{T} 1_{Y_{t} \in B} d t \quad \text { for all } T<\tau \text { and all Borel } B \subseteq E .
$$

For compact $K \subset M_{1}(E)$, it follows that for all $y \in E$ [Donsker and Varadhan (1976), Section 7],

$$
\limsup _{T \uparrow \infty} \frac{1}{T} \log \hat{P}^{\lambda, y}\left(\mu_{T} \in K, T<\tau\right) \leq-\inf _{\mu \in K} I^{\lambda}(\mu) .
$$

Furthermore, the following facts hold.

Lemma 33. Let Assumptions 1, 11 and 12 hold. If there exists a $\mu^{*} \in$ $M_{1}(E)$ such that $I^{\lambda}\left(\mu^{*}\right)=0$, then for all $y \in E, \hat{P}^{\lambda, y}(\tau=\infty)=1$ and $\mu^{*}$ possesses a $C^{2, \gamma}(E, \mathbb{R})$ density $g^{*}$, such that $\tilde{L}^{\lambda} g^{*}=0$, where $\tilde{L}^{\lambda}$ is the formal adjoint to $\hat{L}^{\lambda}$.

Proof. That $\hat{P}^{\lambda, y}(\tau=\infty)=1$ for all $y \in E$ follows by repeating the argument in Lemma 3.5 up to, and through the point where $\hat{P}^{\lambda, y}(\tau=\infty)=1$ for $\mu^{*}$ a.e. $y \in E$. By Pinsky [(1995), Theorem 1.15.1], the same conclusion can be extended to all $y \in E$.

As for the second statement, the argument in Lemma 3.6 can be repeated up to and including the point where it is shown that $I^{\lambda}\left(\mu^{*}\right)=0$ implies $\int_{E} \hat{L}^{\lambda} w(x) \mu^{*}(d x)=0$ for all $w \in C_{0}^{\infty}(E, \mathbb{R})$. Thus the conclusions follow from Pinsky (1995), page 181. In particular, $\int_{E} g^{*} d y=1<\infty$.

Proof of Theorem 18. As in the proof of Theorem 13, the proof of Theorem 18 adapts the results in Kaise and Sheu (2006) to the present setting, now extending Theorem 3.7 and Theorem 3.8. Theorem 3.7 yields 
that for $\lambda=\lambda_{c}, Y$ is $\left(\hat{P}^{y}\right)_{y \in E}$-positive recurrent, while Theorem 3.8 states that the solution $v_{c}$ corresponding to $\lambda_{c}$ is unique up to an additive constant. Theorem 3.8 carries over with only notational changes, in the light of (91) and Lemma 30.

Theorem 3.7 is composed of three parts. The first part (Proposition 3.3) states that if $Y$ is transient under $\left(\hat{P}^{\lambda, y}\right)_{y \in E}$, then there exists $\alpha>0$ such that for all $f \in C_{0}(E), f \geq 0, y \in E$, and $T$ large enough

$$
E_{\hat{P}^{\lambda}}^{y}\left[f\left(Y_{T}\right), T<\tau\right] \leq C(y) e^{-\alpha T} .
$$

The second part uses the first part to show that $Y$ is recurrent under $\left(\hat{P}^{\lambda_{c}, y}\right)$. The third part states that $Y$ is actually positive recurrent. The argument used to prove the second part (recurrence) carries over unchanged, hence, details are provided on the first and third parts.

As for the first part (Proposition 3.3) assume that $Y$ is transient under $\left(\hat{P}^{\lambda, y}\right)_{y \in E}$. Consider the set $\mathcal{C}_{m}=\left\{\mu \in M_{1}(E): \mu\left(E_{l}\right) \geq 1-\delta_{l} \forall l \geq m\right\}$. To construct the constants $\delta_{l}$, set

$$
U_{0}=-\left(V+\frac{1}{2} \nabla w^{\prime} \hat{A} \nabla w+\tilde{b}^{\prime} \nabla w+\frac{1}{2} \operatorname{tr}\left(A D^{2}\right)\right)
$$

for the function $w$ from Assumption 16. Note that by (36),

$$
\lim _{n \uparrow \infty} \inf _{y \in E \backslash E_{n}} U_{0}(y)=\infty .
$$

Let $c$ be as in (91). Set $\beta_{0}=\inf _{y \in E} c\left(U_{0}(y)+\lambda\right)$ and $\beta_{l}=\inf _{y \in E \backslash E_{l}} c\left(U_{0}(y)+\lambda\right)$. Let $M>0$ and set

$$
\delta_{l}=\frac{M+\left|\beta_{0}\right|}{\left|\beta_{0}\right|+\beta_{l}} .
$$

Since $\beta_{l} \uparrow \infty$, it follows that $\delta_{l} \downarrow 0$ and hence, the set $\mathcal{C}_{m}$ is relatively compact (weak topology) for $m$ large enough, so that $\beta_{m}>0$ and $\delta_{m}<1$. Set $\bar{\phi}=$ $\exp (c(w-v))$ where $v$ is such that $M v=\lambda$. Then, repeating the arguments in Proposition 3.3, it follows that for any $f \in C_{0}(E)$ [equation (3.22)],

$$
E_{\hat{P}^{\lambda}}^{y}\left[f\left(Y_{T}\right), T<\tau\right] \leq \sup _{E}|f| \hat{P}^{\lambda, y}\left[\mu_{T} \in \mathcal{C}_{m}, T<\tau\right]+\sup _{E}|f / \bar{\phi}| \bar{\phi}(y) e^{-M t} .
$$

By (93) it follows, after taking $M \uparrow \infty$ that

$$
\limsup _{T \uparrow \infty} \frac{1}{T} \log E_{\hat{P}^{\lambda}}^{y}\left[f\left(Y_{T}\right), T<\tau\right] \leq-\inf _{\mu \in \overline{\mathcal{C}}_{m}} I^{\lambda}(\mu) .
$$

Now, since $I^{\lambda}$ is lower semi-continuous and $\overline{\mathcal{C}}_{m}$ is compact, $-\inf _{\mu \in \overline{\mathcal{C}}_{m}} I^{\lambda}(\mu)=$ $-I^{\lambda}\left(\mu^{*}\right)$ for some $\mu^{*} \in \overline{\mathcal{C}}_{m}$.

It is now shown that $I^{\lambda}\left(\mu^{*}\right)>0$. Suppose, by contradiction, that $I^{\lambda}\left(\mu^{*}\right)=0$ and that $Y$ is transient under $\left(\hat{P}^{\lambda, y}\right)_{y \in E}$. Since $I^{\lambda}\left(\mu^{*}\right)=0$, Lemma 33 implies that:

(a) $\hat{P}^{\lambda, y}[\tau=\infty]=1$ for all $y \in E$. 
(b) With $\tilde{L}^{\lambda}$ denoting the adjoint operator to $\hat{L}^{\lambda}$, there exists a $C^{2, \gamma}(E, \mathbb{R})$ positive function $g^{*}$ such that $\tilde{L}^{\lambda} g^{*}=0$ and $\int_{E} g^{*}(x) d x=1$.

However, Pinsky [(1995), Corollary 4.9.4] implies that if $Y$ is transient un$\operatorname{der}\left(\hat{P}^{\lambda, y}\right)_{y \in E}$ and there exists some $\tilde{\phi} \in C^{2, \gamma}(E, \mathbb{R}), \tilde{\phi}>0$ which satisfies $\tilde{L}^{\lambda} \tilde{\phi}=0$, then $\int_{E} \tilde{\phi} d y<\infty$ implies $\hat{P}^{\lambda, y}[\tau<\infty]>0$ for all $y \in E$. This conclusion contradicts (a) above. Thus, $I^{\lambda}\left(\mu^{*}\right)>0$ and, in view of (95), the inequality in (94) holds for any $0<\alpha<I^{\lambda}\left(\mu^{*}\right)$ and large enough $T$.

To show the third part [positive recurrence for $Y$ under $\left(\hat{P}^{\lambda, y}\right)_{y \in E}$ ], the same steps as in part one can be repeated to obtain (95), as these steps do not require that $Y$ is transient. Now, if $\inf _{\mu \in \overline{\mathcal{C}}_{m}} I^{\lambda}(\mu)>0$, then for all $f \in C_{0}(E),(94)$ implies that

$$
\int_{0}^{\infty} E_{\hat{P}^{\lambda}}^{y}\left[f\left(Y_{T}\right), T<\tau\right] d T<\infty
$$

in which case $Y$ is transient under $\left(\hat{P}^{\lambda, y}\right)_{y \in E}$ [Pinsky (1995), Chapter 4.2]. But part two implies that $Y$ is recurrent. Thus, there is a $\mu^{*} \in \overline{\mathcal{C}}_{m}$ such that $I^{\lambda}\left(\mu^{*}\right)=0$. Since $\mu^{*}$ is a probability measure, ergodicity follows by Lemma 33 .

Proof of Proposition 19. The invariant density for $Y$ under $\left(\hat{P}^{y}\right)_{y \in E}$ is $\tilde{\phi}=\phi_{c}^{2} m_{\nu}$ where $\phi_{c}=\exp \left(v_{c} / \delta\right)$ and $m_{\nu}$ is from (34). Equations (25) and (26) from Theorem 7 become

$$
\begin{aligned}
E_{P}^{y}\left[\left(X_{T}^{\pi}\right)^{p}\right] & =e^{\lambda T+v(y)} E_{\hat{P}}^{y}\left[\phi_{c}\left(Y_{T}\right)^{-\delta}\right], \\
E_{P}^{y}\left[\left(M_{T}^{\eta}\right)^{q}\right]^{1-p} & =e^{\lambda T+v(y)} E_{\hat{P}}^{y}\left[\phi_{c}\left(Y_{T}\right)^{-\delta /(1-p)}\right]^{1-p} .
\end{aligned}
$$

Since $\int_{E} m_{\nu} d y<\infty, \int_{E} \phi_{c}^{2} m_{\nu} d y<\infty$, it follows that

$$
\int_{E} \phi_{c}^{-\delta} \phi_{c}^{2} m_{\nu} d y<\infty, \quad \int_{E} \phi_{c}^{-\delta /(1-p)} \phi_{c}^{2} m_{\nu} d y<\infty
$$

provided that $2-\delta>0$ and $2-\frac{\delta}{1-p}>0$. These conditions are equivalent to those in (39). Thus, by the ergodic result (30) it holds that

$$
\begin{gathered}
\lim _{T \uparrow \infty} E_{\hat{P}}^{y}\left[\phi_{c}\left(Y_{T}\right)^{-\delta}\right]=\int_{E} \phi_{c}^{-\delta} \phi_{c}^{2} m_{\nu} d y \equiv K_{1}, \\
\lim _{T \uparrow \infty} E_{\hat{P}}^{y}\left[\phi_{c}\left(Y_{T}\right)^{-\delta /(1-p)}\right]^{1-p}=\int_{E} \phi_{c}^{-\delta /(1-p)} \phi_{c}^{2} m_{\nu} d y \equiv K_{2}
\end{gathered}
$$

and long-run optimality follows. Furthermore, in light of (19), the conclusion in (40) follows with $K=\frac{1}{p} \log \left(K_{2} / K_{1}\right)$. 


\section{APPENDIX C: PROOFS OF SECTION 4}

Proof of Theorem 22. If $v_{0} \in \mathbb{R}^{k}$ and $v_{1} \in \mathbb{R}^{k \times k}, v_{1}$ symmetric solve (55) and (56), respectively, then $v(y)=v_{0}^{\prime} y-\frac{1}{2} y^{\prime} v_{1} y$ solves (22) for $\lambda$ from (57). Under Assumption 21, the condition (37) holds, hence, Theorems 13 and 18 imply that, if for $v$ the associated process $Y$ is $\hat{P}^{y}$-tight in $\mathbb{R}^{k}$ for each $y \in \mathbb{R}^{k}$, then $v$ is the desired solution. The Riccati equation (56) admits the form

$$
v_{1} \mathbf{B B}^{\prime} v_{1}-v_{1} \mathbf{A}-\mathbf{A}^{\prime} v_{1}-\mathbf{C}^{\prime} \mathbf{C}=0
$$

with

$$
\mathbf{B}=\left(A-q \Upsilon^{\prime} \Sigma^{-1} \Upsilon\right)^{1 / 2} ; \quad \mathbf{A}=-\left(b+q \Upsilon^{\prime} \Sigma^{-1} \mu_{1}\right) ; \quad \mathbf{C}=\sqrt{q} \sigma^{-1} \mu_{1},
$$

where $\mathbf{B}$ is assumed to be the unique symmetric positive definite square root of $A-q \Upsilon^{\prime} \Sigma^{-1} \Upsilon$. C is a real valued matrix when $p<0$. For a real valued square matrix $M$, write $M>0$ if $M+M^{\prime}$ is strictly positive definite. If $M>0$, then the real part of each of its eigenvalues is strictly positive. To see this, let $x, \lambda$ such that $M x=\lambda x$. Then (with $\bar{x}$ denoting the complex conjugate of $x$ )

$$
0<\bar{x}^{\prime}\left(M+M^{\prime}\right) x=\bar{x}^{\prime} \lambda x+\bar{\lambda} \bar{x}^{\prime} x=2|x|^{2} \operatorname{Re}[\lambda] .
$$

From Abou-Kandil et al. [(2003), Lemma 2.4.1], if there exist two matrices $F_{1} \in \mathbb{R}^{k \times k}$ and $F_{2} \in \mathbb{R}^{n \times k}$ such that $\mathbf{A}-\mathbf{B} F_{1}<0$ and $\mathbf{A}^{\prime}-\mathbf{C}^{\prime} F_{2}<0$, then there exists a unique solution $v_{1}$ such that $\mathbf{A}-\mathbf{B B}^{\prime} v_{1}<0$. Since $b>0$ and $p<0$, the choice of $F_{1}=-q \mathbf{B}^{-1} \Upsilon^{\prime} \Sigma^{-1} \mu_{1}$ and $F_{2}=-\sqrt{q} \rho a^{\prime}$ suffices. The condition $\mathbf{A}-\mathbf{B B}^{\prime} v_{1}<0$ yields

$$
-\left(\left(b+q \Upsilon^{\prime} \Sigma^{-1} \mu_{1}\right)+\left(A-q \Upsilon^{\prime} \Sigma^{-1} \Upsilon\right) v_{1}\right)<0 .
$$

Therefore,

$$
\begin{aligned}
& \left(v_{1}\left(A-q \Upsilon^{\prime} \Sigma^{-1} \Upsilon\right)+\left(b+q \Upsilon^{\prime} \Sigma^{-1} \mu_{1}\right)^{\prime}\right) \\
& \quad=\left(\left(b+q \Upsilon^{\prime} \Sigma^{-1} \mu_{1}\right)+\left(A-q \Upsilon^{\prime} \Sigma^{-1} \Upsilon\right) v_{1}\right)^{\prime}>0
\end{aligned}
$$

has eigenvalues with strictly positive real part and is invertible. Thus $v_{0}$ from $(55)$ is well defined. It remains to prove that $\left(Y_{t}\right)_{t \geq 0}$ is $\hat{P}^{y}$-tight in $\mathbb{R}^{k}$. Under $\hat{P}^{y}, Y$ has the dynamics

$$
\begin{aligned}
d Y_{t}=\left(-\left(\left(b+q \Upsilon^{\prime} \Sigma^{-1} \mu_{1}\right)+\left(A-q \Upsilon^{\prime} \Sigma^{-1} \Upsilon\right) v_{1}\right) Y_{t}\right. \\
\left.-q \Upsilon^{\prime} \Sigma^{-1} \mu_{0}+\left(A-q \Upsilon^{\prime} \Sigma^{-1} \Upsilon\right) v_{0}\right) d t+a d W_{t}
\end{aligned}
$$

Setting

$$
\begin{aligned}
& \mathbf{D}=\left(\left(b+q \Upsilon^{\prime} \Sigma^{-1} \mu_{1}\right)+\left(A-q \Upsilon^{\prime} \Sigma^{-1} \Upsilon\right) v_{1}\right) \\
& \mathbf{E}=\mathbf{D}^{-1}\left(-q \Upsilon^{\prime} \Sigma^{-1} \mu_{0}+\left(A-q \Upsilon^{\prime} \Sigma^{-1} \Upsilon\right) v_{0}\right)
\end{aligned}
$$


the dynamics takes the form

$$
d Y_{t}=\mathbf{D}\left(\mathbf{E}-Y_{t}\right) d t+a d W_{t},
$$

where $\mathbf{D} \in \mathbb{R}^{k \times k}$ is such that $\mathbf{D}>0$ and $\mathbf{E} \in \mathbb{R}^{k}$. For $Z_{t}=Y_{t}-\mathbf{E}$ it follows that

$$
d Z_{t}=-\mathbf{D} Z_{t} d t+a d W_{t}
$$

Since

$$
\left|Y_{t}\right|^{2} \leq 2|\mathbf{E}|^{2}+2\left|Z_{t}\right|^{2}
$$

is suffices to show that $\left(Z_{t}\right)_{t \geq 0}$ is $\hat{P}^{y}$-tight in $\mathbb{R}^{k}$, which follows because any compact set is contained in a closed ball around the origin, and by (97) if $Z_{t}$ is in a closed ball around 0 , then so is $Y_{t}$. Using the methods derived in Bhattacharya (1978) it follows that under $\left(\hat{P}^{y}\right)_{y \in E}, Z$ is positive recurrent. To show this, let $\lambda^{*}$ and $\lambda_{*}$ denote the maximum and minimum eigenvalues of $A=a a^{\prime}$ and let $\theta_{*}$ denote the minimum eigenvalue of $\mathbf{D}+\mathbf{D}^{\prime}$. Since $\mathbf{D}>0$ and by Assumption 21, $A>0$ it follows that $\lambda_{*}>0$ and $\theta_{*}>0$. Furthermore,

$$
\inf _{x:|x|=1} x^{\prime} A x=\lambda_{*} \quad \inf _{x:|x|=1} x^{\prime} \mathbf{D} x=\theta_{*} .
$$

Thus, with the notation of Pinsky [(1995), Theorem 6.2], $\underline{\alpha}(r)=\lambda_{*}$ and

$$
\bar{\beta}(r)=\sup _{x:|x|=1} \frac{\operatorname{tr}(A)-x^{\prime} A x-2 r^{2} x^{\prime} \mathbf{D} x}{x^{\prime} A x} \leq \frac{\operatorname{tr}(A)-\lambda_{*}-2 r^{2} \theta_{*}}{\lambda^{*}},
$$

where the last inequality follows for $r$ large enough so that the numerator is negative. Therefore, Pinsky [(1995), Theorem 6.2] applies and $Z$ is positive recurrent.

Proof of Proposition 23. In light of Theorems 22 and 9, it suffices to show that for $p<0$ the following quantity is bounded as a function of $y$ :

$$
\begin{aligned}
\left(p r_{0}-\lambda-\frac{1}{2} q\left(\nu_{0}^{\prime}\right.\right. & \left.+b \nu_{1}^{\prime} y\right)\left(\nu_{0}+b \nu_{1} y\right) \\
& \left.+\frac{1}{2} q \rho^{\prime} \rho\left(v_{0}-v_{1} y\right)^{2}\right) e^{-v_{0} y+(1 / 2) v_{1} y^{2}} .
\end{aligned}
$$

Note that, for $p<0, \Theta$ in (62) satisfies

$$
\Theta>\left(1+q \rho^{\prime} \nu_{1}\right)^{2} .
$$

Therefore,

$$
v_{1}=b \delta\left(\sqrt{\Theta}-\left(1+q \rho^{\prime} \nu_{1}\right)\right)>0
$$

and (98) is bounded over $\mathbb{R}$ only if the quadratic term is negative

$$
\frac{1}{2} q v_{1}^{2} \rho^{\prime} \rho-\frac{1}{2} q b^{2} \nu_{1}^{\prime} \nu_{1}<0 .
$$


But

$$
\begin{aligned}
\frac{1}{2} q v_{1}^{2} \rho^{\prime} \rho-\frac{1}{2} q b^{2} \nu_{1}^{\prime} \nu_{1}= & \frac{1}{2} q \rho^{\prime} \rho \delta^{2} b^{2}\left(\sqrt{\Theta}-\left(1+q \rho^{\prime} \nu_{1}\right)\right)^{2} \\
& -\frac{1}{2} b^{2} \delta\left(\Theta-\left(1+q \rho^{\prime} \nu_{1}\right)^{2}\right) \\
= & \frac{1}{2} \delta^{2} b^{2}\left(\sqrt{\Theta}-\left(1+q \rho^{\prime} \nu_{1}\right)\right) \\
& \times\left(\left(2 q \rho^{\prime} \rho-1\right) \sqrt{\Theta}-\left(1+q \rho^{\prime} \nu_{1}\right)\right) .
\end{aligned}
$$

From $(65)$, the quantity $\left(2 q \rho^{\prime} \rho-1\right) \sqrt{\Theta}-\left(1+q \rho^{\prime} \nu_{1}\right)$ is negative, while $\frac{1}{2} \delta^{2} b^{2}\left(\sqrt{\Theta}-\left(1+q \rho^{\prime} \nu_{1}\right)\right)$ is positive by $(99)$. Therefore, the leading quadratic term is negative and the result follows.

Proof of Corollary 24. When $\nu_{1}=-\kappa \rho$ condition (65) reduces to

$$
\left(1-2 q \rho^{\prime} \rho\right)\left(1+q \rho^{\prime} \rho\left(\kappa^{2}-2 \kappa\right)\right)^{1 / 2}+\left(1-q \kappa \rho^{\prime} \rho\right)>0 .
$$

Set $x=q \rho^{\prime} \rho$ and consider the continuous function

$$
f(x, \kappa)=(1-2 x)\left(1+x\left(\kappa^{2}-2 \kappa\right)\right)^{1 / 2}+(1-\kappa x)
$$

on $0<x<1, \kappa \in \mathbb{R}$. For a fixed $0<x<1$ consider the implicit equation for $\kappa$ obtained by setting $f(x, \kappa)=0$. For $0<x \leq \frac{1}{4}$ one can show there are no $\kappa \in \mathbb{R}$ such that $f(x, \kappa)=0$. For $\frac{1}{4}<x<1, f(x, \kappa)=0$ only along the curve $\kappa=\frac{2}{4 x-1}$. For a fixed $x$ and large positive $\kappa$

$$
f(x, \kappa) \approx \kappa((1-2 x) \sqrt{x}-x)<0
$$

and for large negative $\kappa$

$$
f(x, \kappa) \approx|\kappa|((1-2 x) \sqrt{x}+x)>0,
$$

therefore, plugging back in $q \rho^{\prime} \rho$ for $x$, for $\frac{1}{4}<q \rho^{\prime} \rho<1$ the restriction $\kappa<$ $\frac{2}{4 q \rho^{\prime} \rho-1}$ is necessary.

Proof of Proposition 25. When $\kappa=1$, by Corollary 24, long-run optimality holds for $0<q \rho^{\prime} \rho \leq \frac{1}{4}$. For $\frac{1}{4}<q \rho^{\prime} \rho<1$ long-run optimality holds if $1<\frac{2}{4 q \rho^{\prime} \rho-1}$ or $q \rho^{\prime} \rho<\frac{3}{4}$. Consider now $q \rho^{\prime} \rho \geq \frac{3}{4}$, which is equivalent to $\delta \geq 4$ since $\delta=\frac{1}{1-q \rho^{\prime} \rho}$. When $\kappa=1$ the solution $v_{1}, v_{0}$ and $\Theta$ simplify considerably to $\Theta=\delta^{-1} ; v_{1}=b(\sqrt{\delta}-1)$; and $v_{0}=q \delta \rho^{\prime} \nu_{0}$. Under $\hat{P}, Y$ has the dynamics

$$
d Y_{t}=-\frac{b}{\sqrt{\delta}} Y_{t} d t+d W_{t} .
$$

For $Y_{0}=y$, it follows that $Y_{t} \sim N\left(\mu_{t}, \sigma_{t}^{2}\right)$ with $\mu_{t}=y e^{-b / \sqrt{\delta} t}$, and $\sigma_{t}^{2}=$ $\frac{\sqrt{\delta}}{2 b}\left(1-e^{-2 b / \sqrt{\delta} t}\right)$. Therefore,

$$
E_{\hat{P}}^{y}\left[e^{-v\left(Y_{t}\right)}\right]=E\left[\exp \left(\mathbf{A} Y_{t}^{2}+\mathbf{B} Y_{t}\right)\right]
$$


where $\mathbf{A}=\frac{b}{2}(\sqrt{\delta}-1), \mathbf{B}=-q \delta \rho^{\prime} \nu_{0}$. For $X \sim N\left(\mu, \sigma^{2}\right)$,

$$
\begin{aligned}
& E\left[e^{\mathbf{A} X^{2}+\mathbf{B} X}\right] \\
& =\left\{\begin{array}{c}
\left(1-2 \mathbf{A} \sigma^{2}\right)^{-1 / 2} \exp \left(\left(1-2 \mathbf{A} \sigma^{2}\right)^{-1}\left(\mu^{2} \mathbf{A}+\mu \mathbf{B}+\frac{1}{2} \sigma^{2} \mathbf{B}^{2}\right)\right), \\
\mathbf{A}<\frac{1}{2 \sigma^{2}}, \\
\quad \mathbf{A} \geq \frac{1}{2 \sigma^{2}} .
\end{array}\right.
\end{aligned}
$$

Therefore, $E_{\hat{P}}^{y}\left[e^{-v\left(Y_{t}\right)}\right]<\infty$ if and only if $\frac{b}{2}(\sqrt{\delta}-1)<\frac{1}{2 \sigma_{t}^{2}}$. This condition reduces to

$$
1+\frac{\sqrt{\delta}}{2}(1-\sqrt{\delta})\left(1-e^{-(2 b / \sqrt{\delta}) t}\right)>0
$$

Note that the left-hand side of (101) is equal to 1 at $t=0$ and monotonically decreasing in $t$ for $\delta>1$. Setting this expression equal to 0 , and solving for $t$ yields

$$
\hat{t}=-\frac{\sqrt{\delta}}{2 b} \log \left(\frac{\sqrt{\delta}(\sqrt{\delta}-1)-2}{\sqrt{\delta}(\sqrt{\delta}-1)}\right) .
$$

If $\sqrt{\delta}(\sqrt{\delta}-1)>2$ or equivalently, $\delta>4$, then $\hat{t}>0$ exists. This proves statement (i) in Proposition 25. If $\delta=4$, the left-hand side of (101) reduces to $e^{-b t}>0$ for all $t>0$. Thus,

$$
E_{\hat{P}}^{y}\left[e^{-v\left(Y_{t}\right)}\right]=\exp \left(\frac{b}{2}\left(t+y^{2}\right)-4 q \rho^{\prime} \nu_{0} e^{b / 2 t} y+\frac{8}{b} q^{2}\left(\rho^{\prime} \nu_{0}\right)^{2}\left(1-e^{-b t}\right) e^{b t}\right) .
$$

On the other hand, for $\delta=4$, it follows that $E_{\hat{P}}^{y}\left[e^{-(1 /(1-p)) v\left(Y_{t}\right)}\right]^{1-p}<\infty$ if

$$
\frac{q}{2}+\frac{1}{2(1-p)} e^{-b t}>0
$$

which is true for all $t>0$ because $\delta=4$ only when $0<q<1$. Thus,

$$
\begin{gathered}
E_{\hat{P}}^{y}\left[e^{-(1 /(1-p)) v\left(Y_{t}\right)}\right]^{1-p} \\
=\left(1-(1-q)\left(1-e^{-b t}\right)\right)^{-(1-p) / 2} \\
\quad \times \exp \left(( 1 - p ) \left(\frac{1}{2} b(1-q) y^{2} e^{-b t}-4 q(1-q) \rho^{\prime} \nu_{0} e^{-b / 2 t} y\right.\right. \\
\left.+\frac{8}{b} q^{2}(1-q)^{2}\left(\rho^{\prime} \nu_{0}\right)^{2}\left(1-e^{-b t}\right)\right) \\
\left./\left(1-(1-q)\left(1-e^{-b t}\right)\right)\right) .
\end{gathered}
$$


If $\nu_{0}=0, E_{\hat{P}}^{y}\left[e^{-v\left(Y_{t}\right)}\right] \sim K e^{\frac{b}{2} t}$ and $E_{\hat{P}}^{y}\left[e^{-(1 /(1-p)) v\left(Y_{t}\right)}\right]^{1-p} \sim q^{-(1-p) / 2}$ for large $t$, so the certainty equivalent loss is bounded by $-\frac{b}{2 p}$, proving (ii) in Proposition 25.

If $\nu_{0} \neq 0$, then $E_{\hat{P}}^{y}\left[e^{-v\left(Y_{t}\right)}\right] \sim e^{K_{1} e^{b t}}$ and $E_{\hat{P}}^{y}\left[e^{-(1 /(1-p)) v\left(Y_{t}\right)}\right]^{1-p} \sim q^{-(1-p) / 2} e^{K_{2}}$ for large $T$, where $K_{1}, K_{2}$ are positive constants. In this case, the certainty equivalent loss diverges to $-\infty$ with speed of the order of $\frac{K_{1}}{t} e^{b t}$. This proves (iii) and the proof is complete.

Proof of Proposition 27. Since $v_{0}, v_{1}$ satisfy $(70), v(y)=v_{0} \log y+$ $v_{1} y$ solves (22). Under $\hat{P}$ the dynamics of $Y$ are

$$
d Y_{t}=\sqrt{\Theta}\left(\frac{\sqrt{\Lambda}+(1 / 2) a^{2}}{\sqrt{\Theta}}-Y_{t}\right) d t+a \sqrt{Y_{t}} d W_{t}
$$

and, as mentioned in Section 4.2, the positivity of $\Theta, \Lambda$ give that $Y$ is $\hat{P}^{y}$ tight in $(0, \infty)$ for each $y \in(0, \infty)$.

Therefore, long-run optimality will follow if the quantity $F$ for $p<0$ from Theorem 9 is bounded over $(0, \infty)$. Specifying to this example, it is necessary to show that

$$
\begin{aligned}
\left(p r_{0}+p r_{1} y\right. & -\lambda-\frac{1}{2} q\left(\nu_{0}^{\prime}+y \nu_{1}\right) \frac{1}{y}\left(\nu_{0}+y \nu_{1}\right) \\
+ & \left.\frac{1}{2} q\left(\frac{v_{0}}{y}+v_{1}\right) a^{2} \rho^{\prime} \rho y\left(\frac{v_{0}}{y}+v_{1}\right)\right) e^{-\left(v_{0} \log y+v_{1} y\right)}
\end{aligned}
$$

is bounded on $y>0$. This expression admits the form

$$
\left(\mathbf{A}+\mathbf{B} y+\mathbf{C} y^{2}\right) y^{-v_{0}-1} e^{-v_{1} y} .
$$

For $v_{0}, v_{1}$ from (70), by (69) it follows that $v_{0}>0, v_{1}<0$ and so (102) will follow only if $\mathbf{A}<0, \mathbf{C}<0$. As for $\mathbf{A}$,

$$
\begin{aligned}
\mathbf{A}= & \frac{1}{2} q a^{2} \rho^{\prime} \rho v_{0}^{2}-\frac{1}{2} q \nu_{0}^{\prime} \nu_{0} \\
= & \frac{1}{2} q a^{2} \rho^{\prime} \rho \frac{\delta^{2}}{a^{4}}\left(\sqrt{\Lambda}-\left(b \theta-q a \rho^{\prime} \nu_{0}-\frac{1}{2} a^{2}\right)\right)^{2} \\
& -\frac{1}{2} \frac{\delta}{a^{2}}\left(\Lambda-\left(b \theta-q a \rho^{\prime} \nu_{0}-\frac{1}{2} a^{2}\right)^{2}\right) \\
= & \frac{1}{2} \frac{\delta^{2}}{a^{2}}\left(\sqrt{\Lambda}-\left(b \theta-q a \rho^{\prime} \nu_{0}-\frac{1}{2} a^{2}\right)\right) \\
& \times\left(\left(2 q \rho^{\prime} \rho-1\right) \sqrt{\Lambda}-\left(b \theta-q a \rho^{\prime} \nu_{0}-\frac{1}{2} a^{2}\right)\right) .
\end{aligned}
$$

From (71)

$$
\left(2 q \rho^{\prime} \rho-1\right) \sqrt{\Lambda}-\left(b \theta-q a \rho^{\prime} \nu_{0}-\frac{1}{2} a^{2}\right)<0 .
$$


Thus, $\mathbf{A}<0$ since by (69)

$$
\frac{1}{2} \frac{\delta^{2}}{a^{2}}\left(\sqrt{\Lambda}-\left(b \theta-q a \rho^{\prime} \nu_{0}-\frac{1}{2} a^{2}\right)\right)>0
$$

As for $\mathbf{C}$,

$$
\begin{aligned}
\mathbf{C} & =\frac{1}{2} q a^{2} \rho^{\prime} \rho v_{1}^{2}+p r_{1}-\frac{1}{2} q \nu_{1}^{\prime} \nu_{1} \\
& =\frac{1}{2} q a^{2} \rho^{\prime} \rho \frac{\delta^{2}}{a^{4}}\left(\left(b+q a \rho^{\prime} \nu_{1}\right)-\sqrt{\Theta}\right)^{2}+\frac{1}{2} \frac{\delta}{a^{2}}\left(\left(b+q a \rho^{\prime} \nu_{1}\right)^{2}-\Theta\right) \\
& =\frac{1}{2} \frac{\delta^{2}}{a^{2}}\left(\left(b+q a \rho^{\prime} \nu_{1}\right)-\sqrt{\Theta}\right)\left(\left(1-2 q \rho^{\prime} \rho\right) \sqrt{\Theta}+\left(b+q a \rho^{\prime} \nu_{1}\right)\right) .
\end{aligned}
$$

From (71)

$$
\left(1-2 q \rho^{\prime} \rho\right) \sqrt{\Theta}+\left(b+q a \rho^{\prime} \nu_{1}\right)>0 .
$$

Thus, $\mathbf{C}<0$, since by (69),

$$
\frac{1}{2} \frac{\delta^{2}}{a^{2}}\left(\left(b+q a \rho^{\prime} \nu_{1}\right)-\sqrt{\Theta}\right)<0 .
$$

Acknowledgments. This paper benefited from the helpful comments of seminar participants at Cornell University, Hitotsubashi University, University of Michigan, Princeton University, University of Texas at Austin, the AMS Meeting in San Diego, the Oberwolfach Workshop on Stochastic Analysis in Finance and the Sixth Seminar on Stochastic Analysis at Ascona. We are indebted to an anonymous referee who helped simplify the proof of the main result and improve its presentation.

\section{REFERENCES}

Abou-Kandil, H., Freiling, G., Ionescu, V. and Jank, G. (2003). Matrix Riccati Equations in Control and Systems Theory. Birkhäuser, Basel. MR1997753

BARBERIS, N. (2000). Investing for the long run when returns are predictable. J. Finance $55225-264$.

Bhattacharya, R. N. (1978). Criteria for recurrence and existence of invariant measures for multidimensional diffusions. Ann. Probab. 6 541-553. MR0494525

Bielecki, T. R. and Pliska, S. R. (1999). Risk-sensitive dynamic asset management. Appl. Math. Optim. 39 337-360. MR1675114

Bielecki, T. R. and Pliska, S. R. (2000). Risk sensitive asset management with transaction costs. Finance Stoch. 4 1-33. MR1790132

Brandt, M. (1999). Estimating portfolio and consumption choice: A conditional Euler equations approach. J. Finance 54 1609-1645.

Brennan, M. and Xia, Y. (2002). Dynamic asset allocation under inflation. The Journal of Finance $\mathbf{5 7}$ 1201-1238.

Buraschi, A., Porchia, P. and Trojani, F. (2010). Correlation risk and optimal portfolio choice. J. Finance 65 393-420. 
Castañeda-Leyva, N. and Hernández-Hernández, D. (2005). Optimal consumptioninvestment problems in incomplete markets with stochastic coefficients. SIAM J. Control Optim. 44 1322-1344 (electronic). MR2178034

ČERnÝ, A. and KALlSEN, J. (2007). On the structure of general mean-variance hedging strategies. Ann. Probab. 35 1479-1531. MR2330978

Cheridito, P., Filipović, D. and Yor, M. (2005). Equivalent and absolutely continuous measure changes for jump-diffusion processes. Ann. Appl. Probab. 15 1713-1732. MR2152242

Cox, J. C. and HuAng, C.-F. (1989). Optimal consumption and portfolio policies when asset prices follow a diffusion process. J. Econom. Theory 49 33-83. MR1024460

Cox, J. C. and Huang, C.-F. (1992). A continuous-time portfolio turnpike theorem. J. Econom. Dynam. Control 16 491-507. MR1172445

Cox, J. C., Ingersoll, J. E. JR. and Ross, S. A. (1985). A theory of the term structure of interest rates. Econometrica $\mathbf{5 3}$ 385-407. MR0785475

Donsker, M. D. and Varadhan, S. R. S. (1975). Asymptotic evaluation of certain Markov process expectations for large time. I. II. Comm. Pure Appl. Math. 28 1-47; ibid. 28 (1975) 279-301. MR0386024

Donsker, M. D. and Varadhan, S. R. S. (1976). Asymptotic evaluation of certain Markov process expectations for large time. III. Comm. Pure Appl. Math. 29 389-461. MR0428471

Donsker, M. D. and Varadhan, S. R. S. (1983). Asymptotic evaluation of certain Markov process expectations for large time. IV. Comm. Pure Appl. Math. 36 183-212. MR0690656

Dumas, B. and Luciano, E. (1991). An exact solution to a dynamic portfolio choice problem under transactions costs. J. Finance $\mathbf{4 6} 577-595$.

Dybvig, P. H., Rogers, L. and BACK, K. (1999). Portfolio turnpikes. Review of Financial Studies 12 165-195.

Feller, W. (1951). Two singular diffusion problems. Ann. of Math. (2) 54 173-182. MR0054814

Fernholz, R. and Karatzas, I. (2005). Relative arbitrage in volatility-stabilized markets. Annals of Finance 1 149-177.

Fleming, W. H. and McEneAney, W. M. (1995). Risk-sensitive control on an infinite time horizon. SIAM J. Control Optim. 33 1881-1915. MR1358100

Fleming, W. H. and Sheu, S. J. (2000). Risk-sensitive control and an optimal investment model. Math. Finance 10 197-213. MR1802598

Fleming, W. H. and Sheu, S. J. (2002). Risk-sensitive control and an optimal investment model. II. Ann. Appl. Probab. 12 730-767. MR1910647

Föllmer, H. and Schachermayer, W. (2007). Asymptotic arbitrage and large deviations. Math. Financ. Econ. 1 213-249. MR2403770

Föllmer, H. and SchweIzer, M. (1991). Hedging of contingent claims under incomplete information. In Applied Stochastic Analysis (London, 1989). Stochastics Monogr. 5 389414. Gordon \& Breach, New York. MR1108430

FritTElli, M. (2000). The minimal entropy martingale measure and the valuation problem in incomplete markets. Math. Finance 10 39-52. MR1743972

Grandits, P. and Rheinländer, T. (2002). On the minimal entropy martingale measure. Ann. Probab. 30 1003-1038. MR1920099

Grossman, S. and Vila, J. (1992). Optimal dynamic trading with leverage constraints. Journal of Financial and Quantitative Analysis 27 151-168.

Grossman, S. and Zhou, Z. (1993). Optimal investment strategies for controlling drawdowns. Math. Finance 3 241-276. 
Hakansson, N. H. (1974). Convergence to isoelastic utility and policy in multiperiod portfolio choice. Journal of Financial Economics 1 201-224.

Hansen, L. P. and Scheinkman, J. A. (2009). Long-term risk: An operator approach. Econometrica 77 177-234. MR2477847

HARrison, J. M. and PliskA, S. R. (1981). Martingales and stochastic integrals in the theory of continuous trading. Stochastic Process. Appl. 11 215-260. MR0622165

Hata, H. and Sekine, J. (2006). Solving long term optimal investment problems with Cox-Ingersoll-Ross interest rates. Adv. Math. Econ. 8 231-255.

He, H. and Pearson, N. D. (1991). Consumption and portfolio policies with incomplete markets and short-sale constraints: The infinite-dimensional case. J. Econom. Theory 54 259-304. MR1122311

Henderson, V. (2005). Analytical comparisons of option prices in stochastic volatility models. Math. Finance 15 49-59. MR2116796

Hobson, D. (2004). Stochastic volatility models, correlation, and the $q$-optimal measure. Math. Finance 14 537-556. MR2092922

Huang, C.-F. and Zariphopoulou, T. (1999). Turnpike behavior of long-term investments. Finance Stoch. 3 15-34. MR1805320

Huberman, G. and Ross, S. (1983). Portfolio turnpike theorems, risk aversion, and regularly varying utility functions. Econometrica 51 1345-1361. MR0736053

JiN, X. (1998). Consumption and portfolio turnpike theorems in a continuous-time finance model. J. Econom. Dynam. Control 22 1001-1026. MR1629559

Kaise, H. and Sheu, S. J. (2004). Risk sensitive optimal investment: Solutions of the dynamical programming equation. In Mathematics of Finance. Contemp. Math. 351 217-230. Amer. Math. Soc., Providence, RI. MR2076543

Kaise, H. and Sheu, S.-J. (2006). On the structure of solutions of ergodic type Bellman equation related to risk-sensitive control. Ann. Probab. 34 284-320. MR2206349

Karatzas, I., Lehoczky, J. P. and Shreve, S. E. (1987). Optimal portfolio and consumption decisions for a "small investor" on a finite horizon. SIAM J. Control Optim. 25 1557-1586. MR0912456

Karatzas, I. and Shreve, S. E. (1991). Brownian Motion and Stochastic Calculus, 2nd ed. Grad. Texts in Math. 113. Springer, New York. MR1121940

Karatzas, I., Lehoczky, J. P., Shreve, S. E. and Xu, G.-L. (1991). Martingale and duality methods for utility maximization in an incomplete market. SIAM J. Control Optim. 29 702-730. MR1089152

Kim, T. and Omberg, E. (1996). Dynamic nonmyopic portfolio behavior. The Review of Financial Studies 9 141-161.

Kramkov, D. and Schachermayer, W. (1999). The asymptotic elasticity of utility functions and optimal investment in incomplete markets. Ann. Appl. Probab. 9 904-950. MR1722287

Kramkov, D. and Sîrbu, M. (2006a). On the two-times differentiability of the value functions in the problem of optimal investment in incomplete markets. Ann. Appl. Probab. 16 1352-1384. MR2260066

Kramkov, D. and Sîrbu, M. (2006b). Sensitivity analysis of utility-based prices and risk-tolerance wealth processes. Ann. Appl. Probab. 16 2140-2194. MR2288717

Kramkov, D. and Sîrbu, M. (2007). Asymptotic analysis of utility-based hedging strategies for small number of contingent claims. Stochastic Process. Appl. 117 1606-1620. MR2438000

KurodA, K. and NAGAI, H. (2002). Risk-sensitive portfolio optimization on infinite time horizon. Stoch. Stoch. Rep. 73 309-331. MR1932164 
Ladyzhenskaya, O. A. and Ural'tseva, N. N. (1968). Linear and Quasilinear Elliptic Equations. Academic Press, New York. MR0244627

Leland, H. (1972). On turnpike portfolios. In Mathematical Models in Investment and Finance (G. SzEgo AND K. Shell, eds.). North-Holland, Amsterdam.

LiU, J. (2007). Portfolio selection in stochastic environments. Review of Financial Studies $201-39$.

Merton, R. (1969). Lifetime portfolio selection under uncertainty: The continuous-time case. Rev. Econom. Statist. 51 247-257.

Munk, C. and Sørensen, C. (2004). Optimal consumption and investment strategies with stochastic interest rates. Journal of Banking and Finance 28 1987-2013.

NAGAI, H. (1996). Bellman equations of risk-sensitive control. SIAM J. Control Optim. 34 74-101. MR1372906

NAGAI, H. (2003). Optimal strategies for risk-sensitive portfolio optimization problems for general factor models. SIAM J. Control Optim. 41 1779-1800 (electronic). MR1972534

NAGAi, H. and Peng, S. (2002). Risk-sensitive dynamic portfolio optimization with partial information on infinite time horizon. Ann. Appl. Probab. 12 173-195. MR1890061

Pham, H. (2002). Smooth solutions to optimal investment models with stochastic volatilities and portfolio constraints. Appl. Math. Optim. 46 55-78. MR1922696

Pнам, H. (2003). A large deviations approach to optimal long term investment. Finance Stoch. 7 169-195. MR1968944

Pinchover, Y. (1992). Large time behavior of the heat kernel and the behavior of the Green function near criticality for nonsymmetric elliptic operators. J. Funct. Anal. 104 54-70. MR1152459

Pinsky, R. G. (1995). Positive Harmonic Functions and Diffusion. Cambridge Stud. Adv. Math. 45. Cambridge Univ. Press, Cambridge. MR1326606

PliskA, S. R. (1986). A stochastic calculus model of continuous trading: Optimal portfolios. Math. Oper. Res. 11 370-382. MR0844013

Rheinländer, T. (2005). An entropy approach to the Stein and Stein model with correlation. Finance Stoch. 9 399-413. MR2211715

Schweizer, M. (1992). Mean-variance hedging for general claims. Ann. Appl. Probab. 2 171-179. MR1143398

SCHWEIZER, M. (1996). Approximation pricing and the variance-optimal martingale measure. Ann. Probab. 24 206-236. MR1387633

Summers, L. (1986). Does the stock market rationally reflect fundamental values? J. Finance 41 591-601.

Wachter, J. (2002). Portfolio and consumption decisions under mean-reverting returns: An exact solution for complete markets. Journal of Financial and Quantitative Analysis 37 63-91.

Zariphopoulou, T. (2001). A solution approach to valuation with unhedgeable risks. Finance Stoch. 5 61-82. MR1807876

\author{
Department of Mathematics and Statistics \\ BOSTON UNIVERSITY \\ 111 Cummington St. \\ Boston, Massachusetts 02215 \\ USA \\ AND \\ School of Mathematical Sciences \\ Dublin City University \\ Glasnevin, Dublin 9 \\ IRELAND \\ E-MAIL: guasoni@bu.edu
}

\author{
Department of Mathematical Sciences \\ Carnegie Mellon University \\ Wean Hall 6113 \\ Pittsbiughi, Pennsylvania 15213 \\ USA \\ E-MAIL: scottrob@andrew.cmu.edu
}

